\title{
On nonhomogeneous elliptic equations with the Hardy-Leray potentials
}

\author{
Huyuan Chen1, Alexander Quaa: 2 and Feng Zhou 3 \\ ${ }^{1}$ Department of Mathematics, Jiangxi Normal University, \\ Nanchang, Jiangxi 330022, PR China \\ ${ }^{2}$ Departamento de Matemática, Universidad Técnica Federico, Santa María \\ Casilla V-110, Avda. España 1680, Valparaíso, Chile \\ ${ }^{3}$ Center for PDEs and Department of Mathematics, East China Normal University, \\ Shanghai, 200241, PR China
}

\begin{abstract}
In this paper, we present some suitable distributional identities of solutions for nonhomogeneous elliptic equations involving the Hardy-Leray potentials and study qualitative properties of the solutions to the corresponding nonhomogeneous problems in this distributional sense. We address some applications on the nonexistence of some nonhomogeneous problems with the Hardy-Leray potentials and the nonexistence principle eigenvalue with some indefinite potentials.
\end{abstract}

Keywords: Distributional identity, Hardy-Leray potential, Isolated singularity.

MSC2010: 35B40, 35J99.

\section{Introduction}

Throughout this paper, we assume that $\Omega$ is an open domain containing the origin in $\mathbb{R}^{N}$ with $N \geq 2, \mu \geq \mu_{0}:=-\frac{(N-2)^{2}}{4}$ and $\mathcal{L}_{\mu}:=-\Delta+\frac{\mu}{|x|^{2}}$. The elliptic operator $\mathcal{L}_{\mu}$ involves the inverse square potential, which is also called as Hardy potential, and related semilinear elliptic problems have been done mainly by variational methods [14, 19, 20, 24], due to the Hardy type inequalities, see the references [1, 10, 12, 26]. A direct extension of definition for the distributional solution to the Hardy problem $\mathcal{L}_{\mu} u=g$ in $\Omega$ may be proposed as

$$
\int_{\Omega} u \mathcal{L}_{\mu} \xi d x=\int_{\Omega} g \xi d x, \quad \forall \xi \in C_{c}^{\infty}(\Omega)
$$

where $g$ is a nonlinearity of $x$ and $u$. When $N \geq 3$ and $\mu \in\left[\mu_{0}, 0\right)$, 7, 20] make use of this notation of distributional solution to show the existence of distributional solutions of (1.1) for particular nonlinearity depending on $u$ under some restriction for $\mu$. Later on, the authors in [22] considered the related Hardy problem in the distributional sense of (1.1) replaced $C_{c}^{\infty}(\Omega)$ by $C_{c}^{\infty}(\Omega \backslash\{0\})$ in some range of $\mu$. Chaudhuri and Cîrstea in [16], Cîrstea in [18] classified the isolated singular classical solution of $\mathcal{L}_{\lambda} u+b(x) h(u)=0$ in $\Omega \backslash\{0\}$, where both $b$ and $h$ consist of regularly varying and slowly varying parts (see their definitions in $\S 1.2 .2$ of [18]). There a solution is considered as a $C^{1}(\Omega \backslash\{0\})$-solution in the sense of distributions in $\Omega \backslash\{0\}$, that is,

$$
\int_{\Omega} \nabla u \nabla \varphi d x-\int_{\Omega} \frac{\lambda}{|x|^{2}} u \varphi d x+\int_{\Omega} b(x) h(u) \varphi d x=0, \quad \forall \varphi \in C_{c}^{1}(\Omega \backslash\{0\})
$$

\footnotetext{
${ }^{1}$ chenhuyuan@yeah.net

2 alexander.quaas@usm.cl

3 fzhou@math.ecnu.edu.cn
} 
holds. Recall that a solution $u$ has a "removable" singularity at the origin if $u$ can be extended to a $C^{1}$-solution in $\mathcal{D}^{\prime}(\Omega)$. Another important attempt is done in [20] to consider the consider the solutions of semilinear Hardy equation in classical sense, in variational sense and in a distributional sense. This subject has been extended into the elliptic equation with Hardy potential with singularities on the boundary, for instance, [4, 23, 27, 37, 36, 31] and the references therein.

However, we observe that the distributional identity (1.2) loses the information at the origin in the case that the test functions are in $C_{c}^{\infty}(\Omega \backslash\{0\})$, and on the other hand, it does not work even for the fundamental solutions by the test functions in $C_{c}^{\infty}(\Omega)$ if $\mu>0$ large enough when we consider the distributional identity (1.1), because of high singularity at the origin of the corresponding fundamental solutions. Furthermore, it loses the huge convenience in dealing with semi-linear elliptic equations involving the Laplacian from the expression of singularities by Dirac mass, for instances [9, 34, 39], where the authors obtained classical solutions of $-\Delta u=f$ with precise isolated singularities by dealing with $-\Delta u=f+k \delta_{0}$ in models: $f$ being nonhomogeneous term, $f(u)=u^{p}$ or $f(u)=u^{p}-u$.

Therefore, it is important for theoretical advances and also for application's interests to obtain an improved version of distributional idnetity (1.1). To this end, we start our analysis form the fundamental solutions of Hardy operators. It is known that when $\mu \geq \mu_{0}$, the problem

$$
\mathcal{L}_{\mu} u=0 \quad \text { in } \quad \mathbb{R}^{N} \backslash\{0\}
$$

has two branches of radially symmetric solutions with the explicit formulas that

$$
\Phi_{\mu}(x)=\left\{\begin{array}{ll}
|x|^{\tau_{-}(\mu)} & \text { if } \quad \mu>\mu_{0}, \\
-|x|^{\tau_{-}(\mu)} \ln |x| & \text { if } \quad \mu=\mu_{0}
\end{array} \quad \text { and } \quad \Gamma_{\mu}(x)=|x|^{\tau_{+}(\mu)},\right.
$$

where

$$
\tau_{-}(\mu)=-\frac{N-2}{2}-\sqrt{\mu-\mu_{0}} \text { and } \tau_{+}(\mu)=-\frac{N-2}{2}+\sqrt{\mu-\mu_{0}} .
$$

Here $\tau_{-}(\mu)$ and $\tau_{+}(\mu)$ are two roots of $\mu-\tau(\tau+N-2)=0$. Moreover, $\Phi_{\mu}$ is a regular solution of (1.3) in the sense that $\mu|\cdot|^{-2} \Phi_{\mu}(\cdot) \in L_{l o c}^{1}\left(\mathbb{R}^{N}\right)$ and we have that

$$
\mathcal{L}_{\mu} \Phi_{\mu}=0 \quad \text { in } \quad \mathcal{D}^{\prime}\left(\mathbb{R}^{N}\right) .
$$

Remark that the mentioned fundamental solution of (1.3) with $\mu=0$ is

$$
\Phi_{0}(x)=\left\{\begin{array}{lll}
|x|^{2-N} & \text { if } & N \geq 3 \\
-\ln |x| & \text { if } & N=2
\end{array}\right.
$$

and $\Phi_{0}$ is a distributional solution of

$$
\mathcal{L}_{0} u=c_{N} \delta_{0} \quad \text { in } \quad \mathbb{R}^{\mathrm{N}}
$$

where

$$
c_{N}=\left\{\begin{array}{lll}
(N-2)\left|\mathcal{S}^{N-1}\right| & \text { if } & N \geq 3, \\
2 \pi & \text { if } \quad N=2
\end{array}\right.
$$

and $\mathcal{S}^{N-1}$ is the sphere of the unit ball in $\mathbb{R}^{N}$ and $\left|\mathcal{S}^{N-1}\right|$ is the area of the unit sphere. We observe that the second branch of fundamental solution $\Gamma_{0} \equiv 1$ for $\mu=0$, which is always omitted in the study of semilinear elliptic equation. Without special stating, we always assume that $\mu \geq \mu_{0}$.

Our motivation of this paper is to provide suitable distributional identities for Hardy problems and to answer the basic question whether it is able to express the isolated singularities by the Dirac mass, in particular, to find out some distributional identity to distinguish the fundamental solutions of $\mathcal{L}_{\mu}$.

Our first result on the distributional identities of the fundamental solutions can be stated as follows: 
Theorem 1.1 Let $d \mu(x)=\Gamma_{\mu}(x) d x$ and

$$
\mathcal{L}_{\mu}^{*}=-\Delta-\frac{2 \tau_{+}(\mu)}{|x|^{2}} x \cdot \nabla
$$

Then

$$
\int_{\mathbb{R}^{N}} \Phi_{\mu} \mathcal{L}_{\mu}^{*}(\xi) d \mu=c_{\mu} \xi(0), \quad \forall \xi \in C_{c}^{1.1}\left(\mathbb{R}^{N}\right)
$$

where

$$
c_{\mu}= \begin{cases}2 \sqrt{\mu-\mu_{0}}\left|\mathcal{S}^{N-1}\right| & \text { if } \quad \mu>\mu_{0}, \\ \left|\mathcal{S}^{N-1}\right| & \text { if } \quad \mu=\mu_{0} .\end{cases}
$$

Here and in what follows, we always take the notation

$$
d \mu(x)=\Gamma_{\mu}(x) d x \quad\left(\text { or } d \mu=\Gamma_{\mu} d x\right)
$$

The distributional identity (1.8) is derived from the observation that $\mathcal{L}_{\mu}\left(\Gamma_{\mu} \xi\right)=\Gamma_{\mu} \mathcal{L}_{\mu}^{*}(\xi)$. In the $d \mu$-distributional sense, $\mathcal{L}_{\mu}^{*}$ is the duality operator of $\mathcal{L}_{\mu}$ and we may say that the fundamental solution $\Phi_{\mu}$ is a $d \mu$-distributional solution of

$$
\mathcal{L}_{\mu} \Phi_{\mu}=c_{\mu} \delta_{0} \quad \text { in } \quad \mathcal{D}^{\prime}\left(\mathbb{R}^{\mathrm{N}}\right)
$$

which coincides (1.6) when $\mu=0$, since $d \mu(x)=d x$ in this case and $\mathcal{L}_{0}^{*}=\mathcal{L}_{0}=-\Delta$. Furthermore, an alternative form of $\mathcal{L}_{\mu}^{*}$ could be given as

$$
\mathcal{L}_{\mu}^{*}(\xi)=-\Gamma_{\mu}^{-1} \operatorname{div}\left(\Gamma_{\mu} \nabla \xi\right),
$$

which plays an essential role in the study of heat kernel for the Hardy operator, see [25, 35. We remark that when $\mu=0, c_{\mu}$ coincides with the coefficient $c_{N}$, while for $\mu=\mu_{0}$ there is a jump for the parameter $c_{\mu}$.

It is known that the operator $\mathcal{L}_{\mu}$ with $\mu \in\left[\mu_{0},+\infty\right) \backslash\{0\}$, is self-adjoint in the normal distributional sense. However, identity (1.8) gives a new distributional sense with respect to a specific weighted measure, which makes the operator $\mathcal{L}_{\mu}$ not self-adjoint. To the best of our knowledge, identity (1.8) is new.

We next continue to extend this identity for bounded domains and apply it for the classification of isolated singularities of nonhomogeneous Hardy problem. Given a bounded $C^{2}$ domain $\Omega$ containing the origin, the Hardy problem

$$
\left\{\begin{array}{c}
\mathcal{L}_{\mu} u=0 \quad \text { in } \quad \Omega \backslash\{0\}, \\
u=0 \quad \text { on } \quad \partial \Omega \\
\lim _{x \rightarrow 0} u(x) \Phi_{\mu}^{-1}(x)=1
\end{array}\right.
$$

has solution $G_{\mu}$ with isolated singularity as $\Phi_{\mu}$ at origin, i.e.

$$
\lim _{|x| \rightarrow 0} \frac{G_{\mu}(x)}{\Phi_{\mu}(x)}=1
$$

Then we have the following distributional identity.

Theorem 1.2 Let $G_{\mu}$ be the solution of (1.10) verifying (1.11), then

$$
\int_{\Omega} G_{\mu} \mathcal{L}_{\mu}^{*}(\xi) d \mu=c_{\mu} \xi(0), \quad \forall \xi \in C_{0}^{1.1}(\Omega)
$$


A deeper knowledge of distributional identities allows us to draw a complete picture of the existence, non-existence and the singularities for the nonhomogeneous problem

$$
\left\{\begin{array}{rll}
\mathcal{L}_{\mu} u=f & \text { in } & \Omega \backslash\{0\}, \\
u=0 & \text { on } & \partial \Omega
\end{array}\right.
$$

where $f: \bar{\Omega} \backslash\{0\} \mapsto \mathbb{R}$ is a Hölder continuous locally in $\bar{\Omega} \backslash\{0\}$.

Motivated by (1.8) and (1.12), we shall classify the isolated singularities of (1.13) by building the connection with

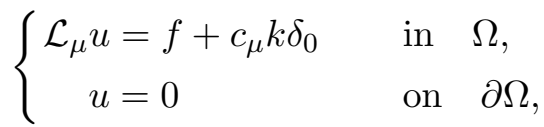

in the $d \mu$-distributional sense that $u \in L^{1}(\Omega, d \mu)$,

$$
\int_{\Omega} u \mathcal{L}_{\mu}^{*}(\xi) d \mu=\int_{\Omega} f \xi d \mu+c_{\mu} k \xi(0), \quad \forall \xi \in C_{0}^{1.1}(\Omega),
$$

where $k \in \mathbb{R}$. Note that the solution $G_{\mu}$ of (1.10) verifying (1.11), from Theorem 1.2, is a $d \mu$ distributional solution of

$$
\left\{\begin{aligned}
\mathcal{L}_{\mu} u & =c_{\mu} \delta_{0} & & \text { in } \quad \Omega, \\
u & =0 & & \text { on } \quad \partial \Omega .
\end{aligned}\right.
$$

For nonhomogeneous problem (1.13), we have the following results.

Theorem 1.3 Let $f$ be a function in $C_{l o c}^{\gamma}(\bar{\Omega} \backslash\{0\})$ for some $\gamma \in(0,1)$.

(i) Assume that

$$
f \in L^{1}(\Omega, d \mu) \quad \text { i. e. } \quad \int_{\Omega}|f| d \mu<+\infty,
$$

then for any $k \in \mathbb{R}$, problem (1.14) admits a unique weak solution $u_{k}$, which is a classical solution of problem (1.13). Furthermore, if assume more that

$$
\lim _{|x| \rightarrow 0} f(x)|x|^{2-\tau_{-}(\mu)}=0,
$$

then we have the asymptotic behavior

$$
\lim _{x \rightarrow 0} u_{k}(x) \Phi_{\mu}^{-1}(x)=k .
$$

(ii) Assume that $f$ verifies (1.16) and $u$ is a nonnegative solution of (1.13), then $u$ is a weak solution of 1.14) for some $k \geq 0$.

(iii) Assume that $f \geq 0$ and

$$
\lim _{r \rightarrow 0^{+}} \int_{\Omega \backslash B_{r}(0)} f d \mu=+\infty
$$

then problem (1.13) has no nonnegative solutions.

Note that Theorem 1.3 part $(i)$ and $(i i)$ build a one to one connection between problems (1.13) and (1.14) under the assumption (1.16). Together with Theorem 1.3 part (iii), the assumptions of (1.16) is sharp for the existence of weak solution to (1.13). Assumption (1.16) could also be seen in [20], where the authors studied the existence of isolated singular solutions of (1.13) only when $N \geq 3$ and $\mu_{0} \leq \mu<0$. Similar assumptions seem be initially proposed in [2, 3] to deal with semilinear parabolic equation with Hardy potentials.

It worth noting that our weak solution $u_{k}$ of (1.14) could be decomposed into $v_{f}+k G_{\mu}$, where $v_{f}$ is a unique solution of (1.15) with $k=0$ and $G_{\mu}$ is the solution of (1.10) subject to (1.11), and the classification of the isolated singularities of (1.13) is performed by Schwartz Theorem in [40]. 
Furthermore, we enlarge the scope of the inhomogeneity into $f \in L^{1}(\Omega, \rho d \mu)$, where $\rho(x)=$ $\operatorname{dist}(x, \partial \Omega)$ in Section 5.2 and show the existence of $d \mu$-distributional solution of (1.14) by approximating the inhomogeneity by regular functions.

By applying the nonexistence result of Theorem 1.3 (iii), we obtain an Liouville theorem for the nonhomogeneous problem (1.13) even for $\mu<\mu_{0}$ in Section 5.2. Finally, our results could be applied to show the nonexistence of a principle eigenvalue problem with an indefinite potential with singularities as Hardy-Leray potentials.

The rest of the paper is organized as follows. In section 2, we show qualitative properties of the solutions to nonhomogeneous problem with regular nonlinearity and "removable" singularity $\lim _{x \rightarrow 0} u(x) \Phi_{\mu}^{-1}(x)=0$. For the uniqueness, the basic tools are comparison principle in the classical sense and the basic tools Kato's inequality in the $d \mu$ weak sense. Section 3 is devoted to build the distributional identity for the fundamental solutions in $\mathbb{R}^{N}$, and in bounded smooth domain and we build the approximation for the fundamental solutions in the distributional sense of (1.12). Section 4 is addressed to study the qualitative properties of the solutions of (1.13) in the distributional sense and to prove Theorem 1.3. Finally, we give a generalization of $d \mu$-distributional solution and an application to the nonexistence of the principle eigenvalue of Laplacian with zero Dirchlet boundary condition.

\section{Preliminary}

\subsection{Comparison Principle for $\mathcal{L}_{\mu}$ and weak singularity}

In this subsection, we introduce the Comparison Principle for the operator $\mathcal{L}_{\mu}$ which is one basic tool in our analysis.

Lemma 2.1 Let $O$ be a bounded open set in $\mathbb{R}^{N}, L: O \times[0,+\infty) \rightarrow[0,+\infty)$ be a continuous function such that for any $x \in O$,

$$
L\left(x, s_{1}\right) \geq L\left(x, s_{2}\right) \quad \text { if } \quad s_{1} \geq s_{2},
$$

then $\mathcal{L}_{\mu}+L$ with $\mu \geq \mu_{0}$ verifies the Comparison Principle, that is, if

$$
u, v \in C^{1,1}(O) \cap C(\bar{O})
$$

satisfying

$$
\mathcal{L}_{\mu} u+L(x, u) \geq \mathcal{L}_{\mu} v+L(x, v) \quad \text { in } \quad O \quad \text { and } \quad u \geq v \quad \text { on } \quad \partial O,
$$

then

$$
u \geq v \quad \text { in } O .
$$

Proof. Let $w=u-v$ and $w_{-}=\min \{w, 0\}$, then $w \geq 0$ on $\partial O$ by the assumption that $u \geq v$ on $\partial O$ and then $w_{-}=0$ on $\partial O$. We will prove that $w_{-} \equiv 0$. If $O_{-}:=\{x \in O: w(x)<0\}$ is not empty, then it is a bounded $C^{1,1}$ domain in $O$. From Hardy inequality with $\mu \geq \mu_{0}$, for some $C>0$, there holds,

$$
\begin{aligned}
0 & =\int_{O_{-}}\left(-\Delta w_{-}+\frac{\mu}{|x|^{2}} w_{-}\right) w_{-} d x+\int_{O_{-}}[L(x, u)-L(x, v)](u-v)_{-} d x \\
& \geq \int_{O_{-}}\left(\left|\nabla w_{-}\right|^{2}+\frac{\mu}{|x|^{2}} w_{-}^{2}\right) d x \geq C \int_{O_{-}} w_{-}^{2} d x
\end{aligned}
$$

then $w_{-}=0$ in a.e. $O_{-}$, which is impossible with the definition of $O_{-}$.

We remark that when $L(s)=s^{p}$, Lemma 2.1] could be seen in [16, Lemma 2.1]. The following lemma plays an important role in the obtention of uniqueness for classical solution. 
Lemma 2.2 Assume that $\Omega$ is a bounded $C^{2}$ domain and $L$ is a continuous function stated in Lemma 2.1 and satisfying $L(x, 0)=0, \forall x \in \Omega$, then the homogeneous problem

$$
\left\{\begin{aligned}
\mathcal{L}_{\mu} u+L(x, u)=0 & \text { in } \quad \Omega \backslash\{0\}, \\
u=0 & \text { on } \quad \partial \Omega, \\
\lim _{x \rightarrow 0} u(x) \Phi_{\mu}^{-1}(x)=0 &
\end{aligned}\right.
$$

has only zero solution.

Proof. Let $u$ be a solution of (2.1), then for any $\epsilon>0$, there exists $r_{\epsilon}>0$ converging to zero as $\epsilon \rightarrow 0$ such that

$$
u \leq \epsilon \Phi_{\mu} \quad \text { in } \quad \overline{B_{r_{\epsilon}}(0)} \backslash\{0\}
$$

We see that

$$
u=0<\epsilon \Phi_{\mu} \quad \text { on } \quad \partial \Omega,
$$

then by Lemma 2.1, we have that

$$
u \leq \epsilon \Phi_{\mu} \quad \text { in } \quad \Omega \backslash\{0\} .
$$

By the arbitrary of $\epsilon$, we have that $u \leq 0$ in $\Omega \backslash\{0\}$. The same way to obtain that $u \geq 0$ in $\Omega \backslash\{0\}$. This ends the proof.

Now let $\mathcal{L}_{\mu}^{*}$ be defined by (1.7). As a consequence of the above result, an important test function we use often in the $d \mu$ distributional sense is the solution $\xi_{0}$ of

$$
\left\{\begin{aligned}
\mathcal{L}_{\mu}^{*} u=1 & \text { in } \quad \Omega, \\
u=0 & \text { on } \quad \partial \Omega
\end{aligned}\right.
$$

satisfies

$$
0<\xi_{0} \leq c \text { in } \Omega
$$

In fact, without loss of the generality, let $\Omega \subset B_{1}(0)$ and denote $v(r)=1-r^{2}$, then

$$
\mathcal{L}_{\mu}^{*} v=2 N+2 \tau_{+}(\mu)>1 .
$$

So $v$ is a super solution of (2.2). For any $x_{0} \in \Omega$, since $\Omega$ is $C^{2}$ domain, there exists $r_{0}>0$ such that $B_{r_{0}}\left(x_{0}\right) \subset \Omega$. Let $w_{t}(x)=t\left(r_{0}^{2}-\left|x-x_{0}\right|^{2}\right)$, then for some $t>0$ small, $w_{t}$ is a sub solution of (2.2). Then (2.3) follows by Lemma 2.1.

Next, we build the distributional identity for the classical solution of the nonhomogeneous problem with "removable" singularity, i.e. $\lim _{x \rightarrow 0} u(x) \Phi_{\mu}^{-1}(x)=0$.

Lemma 2.3 Assume that $f \in C^{\gamma}(\bar{\Omega})$ for some $\gamma \in(0,1)$, then

$$
\left\{\begin{array}{c}
\mathcal{L}_{\mu} u=f \quad \text { in } \quad \Omega \backslash\{0\}, \\
u=0 \quad \text { on } \quad \partial \Omega, \\
\lim _{x \rightarrow 0} u(x) \Phi_{\mu}^{-1}(x)=0
\end{array}\right.
$$

has a unique solution $u_{f}$ satisfying the distributional identity:

$$
\int_{\Omega} u_{f} \mathcal{L}_{\mu}^{*}(\xi) d \mu=\int_{\Omega} f \xi d \mu, \quad \forall \xi \in C_{0}^{1.1}(\Omega) .
$$


Proof. The uniqueness follows by Lemma 2.2 and the existence could be derived by the Perron's method with the super solution $\bar{u}$ chosen as $s\left(\Gamma_{\mu}(x)-t|x|^{2}\right)$ when $\mu_{0} \leq \mu<2 N, s\left(\Gamma_{\mu}(x)-t|x|^{3}\right)$ when $\mu=2 N$ and $s|x|^{2}$ when $\mu>2 N$, where $t>0$ is chosen such that the function is positive on $\Omega$ and then fix $s>0$ compared with $\|f\|_{L^{\infty}(\Omega)}$, and the sub solution $\underline{u}=-\bar{u}$. We are going to prove the distributional identity.

The case $\mu>\mu_{0}$. Indeed, for $\mu>\mu_{0}$, we can choose $\tau_{0} \in\left(\tau_{-}(\mu), \min \left\{2, \tau_{+}(\mu)\right\}\right)$, and denote

$$
V_{0}(x)=|x|^{\tau_{0}}, \quad \forall x \in \Omega \backslash\{0\}
$$

and

$$
\mathcal{L}_{\mu} V_{0}(x)=c_{\tau_{0}}|x|^{\tau_{0}-2},
$$

where

$$
c_{\tau_{0}}=\mu-\tau_{0}\left(\tau_{0}+N-2\right)>0 .
$$

Since $f$ is bounded, there exists $t_{0}>0$ such that

$$
|f(x)| \leq t_{0} c_{\tau_{0}}|x|^{\tau_{0}-2}, \quad \forall x \in \Omega \backslash\{0\},
$$

then $t_{0} V_{0}$ and $-t_{0} V_{0}$ are super solution and sub solution of (2.4), respectively.

For $n$ large, $\Omega \backslash \overline{B_{\frac{1}{n}}(0)}$ is nonempty and the problem

$$
\left\{\begin{array}{ccc}
\mathcal{L}_{\mu} u=f & \text { in } & \Omega \backslash \overline{B_{\frac{1}{n}}(0)}, \\
u=0 & \text { on } & \partial\left(\Omega \backslash \overline{B_{\frac{1}{n}}(0)}\right)
\end{array}\right.
$$

has a unique classical solution $w_{n}$. By Lemma 2.1, we have that

$$
\left|w_{n}(x)\right| \leq t_{0} V_{0}(x), \quad \forall x \in \Omega \backslash\{0\} .
$$

By standard arguments and regularity theory, taking $u_{\mu}=\lim _{n \rightarrow+\infty} w_{n}$, then $u_{\mu}$ is a classical solution of (2.4) and

$$
\left|u_{\mu}(x)\right| \leq t_{0} V_{0}(x), \quad \forall x \in \Omega \backslash\{0\} .
$$

From Lemma 4.9 in [18] with $h \equiv 1$ (e.g. [43]), we have that

$$
\left|\nabla u_{\mu}(x)\right| \leq c V_{0}(x)|x|^{-1}, \quad \forall x \in \Omega \backslash\{0\} .
$$

Thus, for $\xi \in C_{0}^{1.1}(\Omega)$, multiplying $\Gamma_{\mu} \xi$ in (2.4) and integrating over $\Omega \backslash \overline{B_{r}(0)}$, we have that

$$
\begin{aligned}
\int_{\Omega \backslash \overline{B_{r}(0)}} f \xi d \mu= & \int_{\Omega \backslash \overline{B_{r}(0)}} \mathcal{L}_{\mu}\left(u_{\mu}\right) \xi d \mu=\int_{\Omega \backslash \overline{B_{r}(0)}} u_{\mu} \mathcal{L}_{\mu}^{*}(\xi) d \mu \\
& +\int_{\partial B_{r}(0)}\left(\nabla u_{\mu} \cdot \frac{x}{|x|} \Gamma_{\mu}-\nabla \Gamma_{\mu} \cdot \frac{x}{|x|} u_{\mu}\right) \xi d \omega \\
& -\int_{\partial B_{r}(0)} u_{\mu} \Gamma_{\mu}\left(\nabla \xi \cdot \frac{x}{|x|}\right) d \omega .
\end{aligned}
$$

For $r=|x|>0$ small,

$$
\left|\nabla u_{\mu} \cdot \frac{x}{|x|} \Gamma_{\mu} \xi\right| \leq c\|\xi\|_{L^{\infty}(\Omega)} r^{\tau_{0}-1+\tau_{+}(\mu)} \quad \text { and } \quad\left|\nabla \Gamma_{\mu} \cdot \frac{x}{|x|} u_{\mu} \xi\right| \leq c\|\xi\|_{L^{\infty}(\Omega)} r^{\tau_{0}+\tau_{+}(\mu)-1} .
$$

Since $\tau_{0}+\tau_{+}(\mu)-1>1-N$,

$$
\lim _{r \rightarrow 0^{+}} \int_{\partial B_{r}(0)}\left(\nabla u_{\mu} \cdot \frac{x}{|x|} \Gamma_{\mu}-\nabla \Gamma_{\mu} \cdot \frac{x}{|x|} u_{\mu}\right) \xi d \omega=0 .
$$


For the last term in the right hand side of (2.7), we have that

$$
\left|\int_{\partial B_{r}(0)} u_{\mu} \Gamma_{\mu}\left(\nabla \xi \cdot \frac{x}{|x|}\right) d \omega\right| \leq c_{\mu}\|\xi\|_{C^{1}\left(\mathbb{R}^{N}\right)} r \rightarrow 0 \quad \text { as } \quad r \rightarrow 0^{+} .
$$

Therefore, we have that

$$
\int_{\Omega} u_{\mu} \mathcal{L}_{\mu}^{*}(\xi) d \mu=\int_{\Omega} f \xi d \mu
$$

The case $\mu=\mu_{0}$ and $\mu_{0}<0$. By the linearity of $\mathcal{L}_{\mu_{0}}$, we may assume that $f \in C^{1}(\bar{\Omega})$ is nonnegative. By the Comparison Principle, we have that $u_{\mu} \geq 0$ in $\Omega \backslash\{0\}$.

We claim that $\mu \mapsto u_{\mu}$ is decreasing in $\left[\mu_{0}, 0\right)$. Let $\mu_{1} \geq \mu_{2}>\mu_{0}$, then

$$
f=-\Delta u_{\mu_{1}}+\frac{\mu_{1}}{|x|^{2}} u_{\mu_{1}} \geq-\Delta u_{\mu_{1}}+\frac{\mu_{2}}{|x|^{2}} u_{\mu_{1}},
$$

so $u_{\mu_{1}}$ is a sub solution of (2.4) with $\mu=\mu_{2}$. The monotonicity follows by the Comparison Principle in Lemma 2.2 .

We next construct a uniformly bound for $u_{\mu}$ for $\mu>\mu_{0}$. Let

$$
V(x)=|x|^{\tau_{+}\left(\mu_{0}\right)}-\left(s_{0}|x|\right)^{2}, \quad \forall x \in \Omega \backslash\{0\},
$$

where $s_{0}>0$ and $V>0$ in $\Omega \backslash\{0\}$. We see that

$$
\mathcal{L}_{\mu_{0}} V(x)=\frac{(N+2)^{2}}{4} s_{0}^{2}>0, \quad \forall x \in \Omega \backslash\{0\} .
$$

Then there exists $t_{0}>0$ such that

$$
\mathcal{L}_{\mu_{0}}\left(t_{0} V\right) \geq\|f\|_{L^{\infty}(\Omega)}
$$

By the Comparison Principle, we have that

$$
u_{\mu} \leq t_{0} V \quad \text { in } \quad \Omega \backslash\{0\} .
$$

For $\xi \in C_{0}^{1.1}(\Omega)$, there exists $c>0$ independent of $\mu$ such that

$$
\left|\mathcal{L}_{\mu}^{*}(\xi)\right| \leq c\|\xi\|_{C_{0}^{1.1}(\Omega)}+|\mu|\|\xi\|_{C_{0}^{1}(\Omega)}|x|^{-1} .
$$

From the Dominate Monotonicity Convergence Theorem, there exists $u_{\mu_{0}} \leq t_{0} V$ such that

$$
u_{\mu} \rightarrow u_{\mu_{0}} \quad \text { as } \quad \mu \rightarrow \mu_{0}^{+} \quad \text { a.e. in } \Omega \quad \text { and in } \quad L^{1}\left(\Omega,|x|^{-1} d \mu\right) .
$$

Passing to the limit of (2.8) as $\mu \rightarrow \mu_{0}^{+}$, we obtain that

$$
\int_{\Omega} u_{\mu_{0}} \mathcal{L}_{\mu_{0}}^{*}(\xi) d \mu_{0}=\int_{\Omega} f \xi d \mu_{0}
$$

which ends the proof.

\section{$2.2 \quad$ Kato's inequality}

The following proposition is the Kato's type estimate.

Proposition 2.1 Let $\rho(x)=\operatorname{dist}(x, \partial \Omega)$ and $f \in L^{1}(\Omega, \rho d \mu)$, there exists a unique $d \mu$-distributional solution $u \in L^{1}\left(\Omega,|x|^{-1} d \mu\right)$ of the problem

$$
\left\{\begin{aligned}
\mathcal{L}_{\mu} u=f & \text { in } \quad \Omega \\
u=0 & \text { on } \quad \partial \Omega
\end{aligned}\right.
$$


that is,

$$
\int_{\Omega} u \mathcal{L}_{\mu}^{*}(\xi) d \mu=\int_{\Omega} f \xi d \mu, \quad \forall \xi \in C_{0}^{1.1}(\Omega)
$$

Then for any $\xi \in C_{0}^{1.1}(\Omega)$, $\xi \geq 0$, we have that

$$
\int_{\Omega}|u| \mathcal{L}_{\mu}^{*}(\xi) d \mu \leq \int_{\Omega} \operatorname{sign}(u) f \xi d \mu
$$

and

$$
\int_{\Omega} u_{+} \mathcal{L}_{\mu}^{*}(\xi) d \mu \leq \int_{\Omega} \operatorname{sign}_{+}(u) f \xi d \mu .
$$

Proof. Uniqueness. Let $w$ be a $d \mu$-distributional solution of

$$
\left\{\begin{aligned}
\mathcal{L}_{\mu} w=0 & \text { in } \quad \Omega \\
w=0 & \text { on } \quad \partial \Omega
\end{aligned}\right.
$$

For any Borel subset $O$ of $\Omega$, denote by $\eta_{\omega, n}$ the solution of

$$
\left\{\begin{aligned}
\mathcal{L}_{\mu}^{*} u=\zeta_{n} & \text { in } \quad \Omega, \\
u=0 & \text { on } \quad \partial \Omega
\end{aligned}\right.
$$

where $\zeta_{n}: \bar{\Omega} \mapsto[0,1]$ is a $C^{1}(\bar{\Omega})$ function such that $\zeta_{n} \rightarrow \chi_{O}$ in $L^{\infty}(\Omega)$ as $n \rightarrow \infty$. By Lemma 2.3 , we have that $\int_{\Omega} w \Gamma_{\mu} \zeta_{n} d x=0$. Passing to the limit as $n \rightarrow \infty$, we have that

$$
\int_{O} w \Gamma_{\mu} d x=0
$$

which implies that $w=0$.

Existence and estimate (2.10). For $\sigma>0$, we define an even convex function $\phi_{\sigma}$ as

$$
\phi_{\sigma}(t)=\left\{\begin{array}{lll}
|t|-\frac{\sigma}{2} & \text { if } & |\mathrm{t}| \geq \sigma, \\
\frac{t^{2}}{2 \sigma} & \text { if } & |\mathrm{t}|<\sigma / 2 .
\end{array}\right.
$$

Then for any $t \in \mathbb{R},\left|\phi_{\sigma}^{\prime}(t)\right| \leq 1, \phi_{\sigma}(t) \rightarrow|t|$ and $\phi_{\sigma}^{\prime}(t) \rightarrow \operatorname{sign}(\mathrm{t})$ when $\sigma \rightarrow 0^{+}$. Let $\left\{f_{n}\right\}$ be a sequence of functions in $C^{1}(\bar{\Omega})$ such that

$$
\lim _{n \rightarrow \infty} \int_{\Omega}\left|f_{n}-f\right| \rho d \mu=0
$$

Let $u_{n}$ be the corresponding solution to (2.9) with right-hand side $f_{n}$, then for any $\sigma>0$ and $\xi \in C_{0}^{1.1}(\Omega), \xi \geq 0$, we have that

$$
\begin{aligned}
\int_{\Omega} \phi_{\sigma}\left(u_{n}\right)(-\Delta)\left(\Gamma_{\mu} \xi\right) d x & =\int_{\Omega} \Gamma_{\mu} \xi(-\Delta) \phi_{\sigma}\left(u_{n}\right) d x \\
& =\int_{\Omega} \xi \phi_{\sigma}^{\prime}\left(u_{n}\right)(-\Delta) u_{n} d \mu-\int_{\Omega} \xi \phi_{\sigma}^{\prime \prime}\left(u_{n}\right)\left|\nabla u_{n}\right|^{2} d \mu \\
& \leq \int_{\Omega} \xi \phi_{\sigma}^{\prime}\left(u_{n}\right) f_{n} d \mu-\mu \int_{\Omega} \phi_{\sigma}^{\prime}\left(u_{n}\right) \frac{u_{n}}{|x|^{2}} \xi d \mu
\end{aligned}
$$

Letting $\sigma \rightarrow 0$, we obtain that

$$
\int_{\Omega}\left|u_{n}\right|(-\Delta)\left(\Gamma_{\mu} \xi\right) d x \leq \int_{\Omega} \xi \operatorname{sign}\left(u_{n}\right) f_{n} d \mu-\mu \int_{\Omega} \frac{\left|u_{n}\right|}{|x|^{2}} \xi d \mu,
$$


that is,

$$
\int_{\Omega}\left|u_{n}\right| \mathcal{L}_{\mu}^{*}(\xi) d \mu \leq \int_{\Omega} \xi\left|f_{n}\right| d \mu .
$$

Let $\eta_{1}$ be the solution of (2.2) , taking $\xi=\xi_{0}$, the solution of (2.2), then

$$
\int_{\Omega}\left|u_{n}\right| d \mu \leq c_{8} \int_{\Omega}\left|f_{n}\right| \rho d \mu .
$$

Similarly,

$$
\int_{\Omega}\left|u_{n}-u_{m}\right| d \mu \leq c_{8} \int_{\Omega}\left|f_{n}-f_{m}\right| \rho d \mu .
$$

Therefore, $\left\{u_{n}\right\}$ is a Cauchy sequence in $L^{1}(\Omega, \rho d \mu)$ and its limit $u$ is a $d \mu$-distributional solution of (2.9). Passing to the limit as $n \rightarrow \infty$ in (2.16), we obtain (2.10). Inequality (2.11) is proved by replacing $\phi_{\sigma}$ by $\tilde{\phi}_{\sigma}$ which is zero on $(-\infty, 0)$ and $\phi_{\sigma}$ on $[0, \infty)$.

\section{Distributional identities}

\subsection{Fundamental solution.}

The distributional identity of the fundamental solutions is derived by divergence theorem.

Proof of Theorem 1.1. For $\xi \in C_{c}^{1.1}\left(\mathbb{R}^{N}\right)$, multiply $\Gamma_{\mu} \xi$ in (1.3) and integrate over $\mathbb{R}^{N} \backslash \overline{B_{r}(0)}$, we have that

$$
\begin{aligned}
0= & \int_{\mathbb{R}^{N} \backslash \overline{B_{r}(0)}} \mathcal{L}_{\mu}\left(\Phi_{\mu}\right) \Gamma_{\mu} \xi d x=\int_{\mathbb{R}^{N} \backslash \overline{B_{r}(0)}} \Phi_{\mu} \mathcal{L}_{\mu}^{*}(\xi) d \mu \\
& +\int_{\partial B_{r}(0)}\left(\nabla \Phi_{\mu} \cdot \frac{x}{|x|} \Gamma_{\mu}-\nabla \Gamma_{\mu} \cdot \frac{x}{|x|} \Phi_{\mu}\right) \xi d \omega-\int_{\partial B_{r}(0)} \Phi_{\mu} \Gamma_{\mu}\left(\nabla \xi \cdot \frac{x}{|x|}\right) d \omega
\end{aligned}
$$

For $\mu>\mu_{0}$, we see that

$$
\nabla \Phi_{\mu}(x) \cdot \frac{x}{|x|} \Gamma_{\mu}(x)-\nabla \Gamma_{\mu}(x) \cdot \frac{x}{|x|} \Phi_{\mu}(x)=-2 \sqrt{\mu-\mu_{0}}|x|^{1-N}
$$

and for $r=|x|>0$ small,

$$
|\xi(x)-\xi(0)| \leq\|\nabla \xi\|_{L^{\infty}\left(\mathbb{R}^{N}\right)} r
$$

and

$$
\int_{\partial B_{r}(0)}\left(\nabla \Phi_{\mu} \cdot \frac{x}{|x|} \Gamma_{\mu}-\nabla \Gamma_{\mu} \cdot \frac{x}{|x|} \Phi_{\mu}\right) \xi(0) d \omega=-c_{\mu} \xi(0)
$$

where $c_{\mu}=2 \sqrt{\mu-\mu_{0}}\left|S^{N-1}\right|$ for $\mu>\mu_{0}$.

Elementary estimates show that

$$
\lim _{r \rightarrow 0^{+}} \int_{\mathbb{R}^{N} \backslash \overline{B_{r}(0)}} \Phi_{\mu} \mathcal{L}_{\mu}^{*}(\xi) d \mu=c_{\mu} \xi(0) .
$$

For $\mu=\mu_{0}$, we have that $\tau\left(\mu_{0}\right)=\bar{\tau}\left(\mu_{0}\right)=\frac{2-N}{2}$,

$$
\nabla \Phi_{\mu_{0}} \cdot \frac{x}{|x|} \Gamma_{\mu_{0}}-\nabla \Gamma_{\mu_{0}} \cdot \frac{x}{|x|} \Phi_{\mu_{0}}=-|x|^{1-N}
$$

then

$$
\lim _{r \rightarrow 0} \int_{\partial B_{r}(0)}\left(\nabla \Phi_{\mu_{0}} \cdot \frac{x}{|x|} \Gamma_{\mu_{0}} \xi-\nabla \Gamma_{\mu_{0}} \cdot \frac{x}{|x|} \Phi_{\mu_{0}} \xi\right) d \omega=\left|\mathcal{S}^{N-1}\right| \xi(0)
$$


and

$$
\lim _{r \rightarrow 0} \int_{\partial B_{r}(0)} \Phi_{\mu_{0}} \Gamma_{\mu_{0}}\left(\nabla \xi \cdot \frac{x}{|x|}\right) d \omega=0
$$

Thus, we have that

$$
\lim _{r \rightarrow 0^{+}} \int_{\mathbb{R}^{N} \backslash \overline{B_{r}(0)}} \Phi_{\mu_{0}} \mathcal{L}_{\mu_{0}}^{*}(\xi) d \mu_{0}=c_{\mu_{0}} \xi(0)
$$

This ends the proof.

Remark 3.1 For $\mu \neq 0$, it is not proper to divide into each term like

$$
\lim _{r \rightarrow 0^{+}} \int_{\mathbb{R}^{N} \backslash \overline{B_{r}(0)}} \Phi_{\mu} \mathcal{L}_{\mu}\left(\Gamma_{\mu} \xi\right) d x=\lim _{r \rightarrow 0^{+}} \int_{\mathbb{R}^{N} \backslash \overline{B_{r}(0)}} \Phi_{\mu}(-\Delta)\left(\Gamma_{\mu} \xi\right) d x+\lim _{r \rightarrow 0^{+}} \int_{\mathbb{R}^{N} \backslash \overline{B_{r}(0)}} \mu \frac{\Phi_{\mu} \Gamma_{\mu} \xi}{|x|^{2}} d x
$$

In fact, we see that if $\xi(0)>0$,

$$
\lim _{r \rightarrow 0^{+}} \int_{\mathbb{R}^{N} \backslash \overline{B_{r}(0)}} \frac{\Phi_{\mu} \Gamma_{\mu} \xi}{|x|^{2}} d x=+\infty .
$$

\section{$3.2 d \mu$-distributional solution in bounded domain}

Different from the case in $\mathbb{R}^{N}$, there is only one branch of the fundamental solution for $\mathcal{L}_{\mu} u=\delta_{0}$, subject to the Dirichlet boundary condition.

Proof of Theorem 1.2. The existence follows from Perron's methods with the super and subsolutions $\Phi_{\mu}$ and $\Phi_{\mu}-t \Gamma_{\mu}$ respectively. Here $t$ is taken such that $\Phi_{\mu}-t \Gamma_{\mu} \leq 0$ on $\partial \Omega$. The uniqueness follows from the Comparison Principle.

Let $\eta_{0}:[0,+\infty) \rightarrow[0,1]$ be a decreasing $C^{\infty}$ function such that

$$
\eta_{0}=1 \quad \text { in }[0,1] \text { and } \eta_{0}=0 \text { in }[2,+\infty) .
$$

Take $n_{0} \geq 1$ such that

$$
\frac{1}{n_{0}} \sup \left\{r>0: B_{r}(0) \subset \Omega\right\} \leq \frac{1}{2} .
$$

Denote $\eta_{n_{0}}(r)=\eta_{0}\left(n_{0} r\right)$ for $r \geq 0$ and $w_{1}=\Phi_{\mu} \eta_{n_{0}}$ and $w_{2}=G_{\mu}-\Phi_{\mu} \eta_{n_{0}}$, then

$$
\left\{\begin{array}{l}
\mathcal{L}_{\mu} w_{i}=-\nabla \eta_{n_{0}} \cdot \nabla \Phi_{\mu}-\Phi_{\mu} \Delta \eta_{n_{0}} \quad \text { in } \quad \Omega \backslash\{0\}, \\
w_{i}=0 \quad \text { on } \quad \partial \Omega, \\
\lim _{x \rightarrow 0} w_{i}(x) \Phi_{\mu}^{-1}(x)=2-i,
\end{array}\right.
$$

where $i=1,2$. We see that $-\nabla \eta_{n_{0}} \cdot \nabla \Phi_{\mu}-\Phi_{\mu} \Delta \eta_{n_{0}}$ has compact set in $\overline{B_{\frac{2}{n_{0}}}(0)} \backslash B_{\frac{1}{n_{0}}}(0)$ and then $-\nabla \eta_{n_{0}} \cdot \nabla \Phi_{\mu}-\Phi_{\mu} \Delta \eta_{n_{0}}$ is smooth and bounded.

For $i=1$, following the proof of Theorem 1.1, we have that

$$
\int_{\mathbb{R}^{N}} w_{1} \mathcal{L}_{\mu}^{*}(\xi) d \mu=\int_{\Omega}\left[-\nabla \eta_{n_{0}} \cdot \nabla \Phi_{\mu}-\Phi_{\mu} \Delta \eta_{n_{0}}\right] \xi d \mu+c_{\mu} \xi(0), \quad \forall \xi \in C_{0}^{1.1}(\Omega) .
$$

For $i=2$, it follows by Lemma 2.3 that

$$
\int_{\Omega} w_{2} \mathcal{L}_{\mu}^{*}(\xi) d \mu=\int_{\Omega}\left[-\nabla \eta_{n_{0}} \cdot \nabla \Phi_{\mu}-\Phi_{\mu} \Delta \eta_{n_{0}}\right] \xi d \mu, \quad \forall \xi \in C_{0}^{1.1}(\Omega) .
$$

Together with (3.3),

$$
\int_{\Omega} G_{\mu} \mathcal{L}_{\mu}^{*}(\xi) d \mu=c_{\mu} \xi(0), \quad \forall \xi \in C_{0}^{1.1}(\Omega) .
$$

This completes the proof. 


\subsection{Approximation of the fundamental solution}

In this subsection, we see the approximation of the fundamental solution $G_{\mu}$.

Proposition 3.1 Let $\left\{\delta_{n}\right\}_{n}$ be a sequence of nonnegative $L^{\infty}$-functions such that supp $\delta_{n} \subset B_{r_{n}}(0)$, where $r_{n} \rightarrow 0$ as $n \rightarrow+\infty$ and

$$
\delta_{n} \rightarrow \delta_{0} \quad \text { as } n \rightarrow+\infty \text { in the distributional sense. }
$$

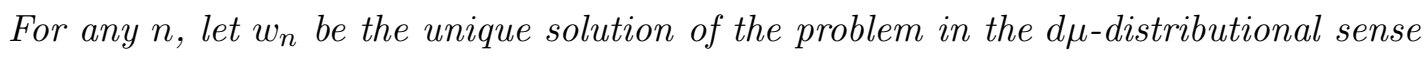

$$
\left\{\begin{array}{c}
\mathcal{L}_{\mu} u=\delta_{n} \quad \text { in } \quad \Omega \backslash\{0\}, \\
u=0 \quad \text { on } \partial \Omega, \\
\lim _{x \rightarrow 0} u(x) \Phi_{\mu}^{-1}(x)=0 .
\end{array}\right.
$$

Then

$$
\lim _{n \rightarrow+\infty} w_{n}(x)=\frac{1}{c_{\mu}} G_{\mu}(x), \quad \forall x \in \Omega \backslash\{0\}
$$

and for any compact set $K \subset \Omega \backslash\{0\}$,

$$
w_{n} \rightarrow \frac{1}{c_{\mu}} G_{\mu} \quad \text { as } n \rightarrow+\infty \text { in } C^{2}(K) .
$$

Proof. From Lemma 2.3 , equation (3.5) has unique solution $w_{n} \geq 0$ satisfying

$$
\int_{\Omega} w_{n} \mathcal{L}_{\mu}^{*}(\xi) d \mu=\int_{\Omega} \delta_{n} \xi d x, \quad \forall \xi \in C_{0}^{1.1}(\Omega) .
$$

In particular, by taking $\xi=\xi_{0}$, the solution of (2.2), we obtain that

$$
\left\|w_{n}\right\|_{L^{1}(\Omega, d \mu)} \leq\left\|\xi_{0}\right\|_{L^{\infty}(\Omega)}\left\|\delta_{n}\right\|_{L^{1}(\Omega)}=\left\|\xi_{0}\right\|_{L^{\infty}(\Omega)} .
$$

For any $r>0$, take $\xi$ with the support in $\Omega \backslash B_{r}(0)$, then $\xi \in C_{c}^{1.1}\left(\overline{\Omega \backslash B_{r}(0)}\right)$,

$$
\int_{\Omega \backslash B_{r}(0)} w_{n} \mathcal{L}_{\mu}^{*}(\xi) d \mu=0
$$

We claim that $w_{n}$ is uniform bounded in $L^{1}\left(\Omega,|x|^{-1} d \mu\right)$. Indeed, for $\sigma>0$, let

$$
\xi_{\sigma}(x)=\left(r_{0}-\phi_{\sigma}(|x|)\right) \eta_{n_{0}}(|x|), \quad \forall x \in \Omega,
$$

where $\phi_{\sigma}$ is defined in (2.14) and $\eta_{0}$ is given in (3.1) and $n_{0}$ is such that $\eta_{n_{0}}$ has compact set in $\Omega$ and $r_{0}>0$ is such that $\Omega \subset B_{r_{0}}(0)$. Then $\xi_{\sigma} \in C_{0}^{1.1}(\Omega)$ and for $x \in B_{\frac{1}{n_{0}}}(0)$, we have that

$$
\mathcal{L}_{\mu}^{*}\left(\xi_{\sigma}\right)(x)=\left\{\begin{array}{lll}
\frac{N-1+\tau_{+}(\mu)}{|x|} & \text { for } & \sigma \leq|x|<1 / n_{0}, \\
\frac{1}{\sigma}+N-1+\tau_{+}(\mu) & \text { for } & |x|<\sigma
\end{array}\right.
$$

and there exists $c>0$ such that

$$
\left|\mathcal{L}_{\mu}^{*}\left(\xi_{\sigma}\right)(x)\right| \leq c, \quad \forall x \in \Omega \backslash B_{\frac{1}{n_{0}}}(0),
$$

where $N-1+\tau_{+}(\mu)>0$ for $\mu \geq \mu_{0}$. Therefore, we have that

$$
\begin{aligned}
\left(N-1+\tau_{+}(\mu)\right) \int_{B_{\frac{1}{n_{0}}}(0) \backslash B_{\sigma}(0)} w_{n}|x|^{-1} d \mu & \leq \int_{B_{\frac{1}{n_{0}}}(0)} w_{n} \mathcal{L}_{\mu}^{*}\left(\xi_{\sigma}\right) d \mu \\
& \leq \int_{\Omega} \delta_{n} \xi_{\sigma} d \mu+c \int_{\Omega \backslash B_{\frac{1}{n_{0}}}(0)} w_{n} d \mu \\
& \leq c
\end{aligned}
$$


where $c>0$ independent of $n$. Passing to the limit as $\sigma \rightarrow 0$, we have that

$$
\int_{B_{\frac{1}{n_{0}}}(0)} w_{n}|x|^{-1} d \mu \leq c^{\prime},
$$

which, together with the fact that

$$
\int_{\Omega \backslash B} w_{\frac{1}{n_{0}}(0)} w_{n}|x|^{-1} d \mu \leq c,
$$

implies that

$$
\int_{\Omega} w_{n}|x|^{-1} d \mu \leq c^{\prime}
$$

that is, $w_{n}$ is uniform bounded in $L^{1}\left(\Omega,|x|^{-1} d \mu\right)$.

From Corollary 2.8 in 45$]$ with $L^{*}=\mathcal{L}_{\mu}^{*}$, which is strictly elliptic in $\Omega \backslash B_{r}(0)$, we have that for $q<\frac{N}{N-1}$

$$
\begin{aligned}
\left\|w_{n} \Gamma_{\mu}\right\|_{W^{1, q}\left(\Omega_{2 r}\right)} & \leq c\left\|\delta_{n}\right\|_{L^{1}\left(\Omega \backslash B_{r}(0)\right)}+c\left\|w_{n}\right\|_{L^{1}\left(\Omega \backslash B_{r}(0), d \mu\right)} \\
& \leq c\left(1+\left\|\xi_{0}\right\|_{L^{\infty}(\Omega)}\right),
\end{aligned}
$$

where $\Omega_{2 r}=\left\{x \in \Omega \backslash B_{2 r}(0): \rho(x)>2 r\right\}$. By the compact embedding

$$
W^{1, q}\left(\Omega_{2 r}\right) \hookrightarrow L^{1}\left(\Omega_{2 r}\right),
$$

up to some subsequence, there exists $w_{\infty} \in W_{l o c}^{1, q}(\Omega) \cap L^{1}(\Omega, d \mu)$ such that

$$
w_{n} \rightarrow w_{\infty} \quad \text { as } n \rightarrow+\infty \quad \text { a.e. in } \Omega \text { and in } L^{1}(\Omega, d \mu)
$$

and it follows by (3.7) that for $\xi \in C_{0}^{1.1}(\Omega)$,

$$
\int_{\Omega} w_{\infty} \mathcal{L}_{\mu}^{*}(\xi) d \mu=\xi(0) .
$$

Furthermore,

$$
\int_{\Omega}\left(w_{\infty}-\frac{1}{c_{\mu}} G_{\mu}\right) \mathcal{L}_{\mu}^{*}(\xi) d \mu=0 .
$$

From the Kato's inequality Lemma 2.1 with $f=0$, we deduce that

$$
w_{\infty}=\frac{1}{c_{\mu}} G_{\mu} \quad \text { a.e. } \Omega .
$$

Proof of (3.6). For any $x_{0} \in \Omega \backslash\{0\}$, let $r_{0}=\frac{1}{4}\left\{\left|x_{0}\right|, \rho\left(x_{0}\right)\right\}$ and

$$
v_{n}=w_{n} \eta,
$$

where $\eta(x)=\eta_{0}\left(\frac{\left|x-x_{0}\right|}{r_{0}}\right)$. There exists $n_{0}>0$ such that for $n \geq n_{0}$,

$$
\operatorname{supp} v_{n} \cap B_{r_{n}}(0)=\emptyset .
$$

Then

$$
\begin{aligned}
-\Delta v_{n}(x) & =-\Delta w_{n}(x) \eta(x)-\nabla w_{n} \cdot \nabla \eta-w_{n} \Delta \eta \\
& =-\nabla w_{n} \cdot \nabla \eta-w_{n} \Delta \eta
\end{aligned}
$$

where $\nabla \eta$ and $\Delta \eta$ are smooth. 
We observe that $w_{n} \in W^{1, q}\left(B_{2 r_{0}}\left(x_{0}\right)\right)$ and $-\nabla w_{n} \cdot \nabla \eta-w_{n} \Delta \eta \in L^{q}\left(B_{2 r_{0}}\left(x_{0}\right)\right)$, then we have that

$$
\left\|v_{n}\right\|_{W^{2, q}\left(B_{r_{0}}\left(x_{0}\right)\right)} \leq c\left\|w_{n}\right\|_{L^{1}(\Omega, d \mu)},
$$

where $c>0$ is independent of $n$. Thus, $-\nabla w_{n} \cdot \nabla \eta-w_{n} \Delta \eta \in W^{1, q}\left(B_{r_{0}}\left(x_{0}\right)\right)$, repeat above process $N_{0}$ steps, for $N_{0}$ large enough, we deduce that

$$
\left\|w_{n}\right\|_{C^{2, \gamma}\left(B \frac{r_{0}}{2^{N_{0}}}\left(x_{0}\right)\right)} \leq c\left\|w_{n}\right\|_{L^{1}(\Omega, d \mu)}
$$

where $\gamma \in(0,1)$ and $c>0$ is independent of $n$. As a conclusion, (3.6) follows by Arzelà-Ascola theorem and Heine-Borel theorem. This ends the proof.

Remark 3.2 Let $\eta_{0}:[0,+\infty) \rightarrow[0,1]$ be a $C^{\infty}$ decreasing function such that $\eta_{0}=1$ in $[0,1]$ and $\eta_{0}=0$ in $[2,+\infty), \delta_{n}(x)=n^{N} \eta_{0}(n|x|)$ for $x \in \mathbb{R}^{N}$. Then

$$
\delta_{n} \rightarrow \delta_{0} \quad \text { in } \mathcal{D}^{\prime}\left(\mathbb{R}^{N}\right) \text { as } n \rightarrow+\infty .
$$

\section{Nonhomogeneous problem}

\subsection{Isolated singularities}

We concerns with the isolated singularities of the solution $v_{f}$ of (1.13) verifying the identity (1.15) with $k=0$. Let $\mathcal{G}_{\mu}$ be the Green's kernel of $\mathcal{L}_{\mu}$ in $\Omega \times \Omega$ with Dirichlet boundary condition. From Theorem 1.2, it holds that for $x \in \Omega \backslash\{0\}$ and $y=0$,

$$
\mathcal{G}_{\mu}(x, 0)=G_{\mu}(x),
$$

which expresses $\delta_{0}$ in the $d \mu$-distributional sense. For $x, y \in \Omega \backslash\{0\}$, we have following estimate of $\mathcal{G}_{\mu}$.

Lemma 4.1 If $\mu_{0}<0$ and $\mu_{0} \leq \mu<0$, we have that for $x, y \in \Omega \backslash\{0\}$,

$$
0<\mathcal{G}_{\mu}(x, y) \leq c\left(|x-y|^{2-N}+\frac{|x|^{\tau_{+}(\mu)}}{|x-y|^{N-2+\tau_{+}(\mu)}}+\frac{|y|^{\tau_{+}(\mu)}}{|x-y|^{N-2+\tau_{+}(\mu)}}+\frac{|x|^{\tau_{+}(\mu)}|y|^{\tau_{+}(\mu)}}{|x-y|^{N-2+2 \tau_{+}(\mu)}}\right)
$$

if $\mu \geq 0$ and $N \geq 3$, we have that for $x, y \in \Omega \backslash\{0\}$,

$$
0<\mathcal{G}_{\mu}(x, y) \leq c \min \left\{|x-y|^{2-N}, \frac{|x|^{\tau_{+}(\mu)}}{|x-y|^{N-2+\tau_{+}(\mu)}}, \frac{|y|^{\tau_{+}(\mu)}}{|x-y|^{N-2+\tau_{+}(\mu)}}, \frac{|x|^{\tau_{+}(\mu)}|y|^{\tau_{+}(\mu)}}{|x-y|^{N-2+2 \tau_{+}(\mu)}}\right\} .
$$

Here and in the following, $|x-y|^{2-N}$ in (4.2) should be replaced by $-\ln |x-y|$ when $N=2$.

Proof. For $\mu_{0}<0$ and $\mu_{0} \leq \mu<0$, it follows from Theorem 3.11 in [25] that the corresponding heat kernel verifies

$$
\begin{aligned}
k_{\mu}(t, x, y) & \leq c \min \left\{\left(1+\frac{|x|}{\sqrt{t}}\right)^{\tau_{+}(\mu)}\left(1+\frac{|y|}{\sqrt{t}}\right)^{\tau_{+}(\mu)},\left(\frac{d(x) d(x)}{|x||y|}\right)^{\tau_{+}(\mu)}\right\} t^{-\frac{N}{2}} e^{-c \frac{|x-y|^{2}}{t}} \\
& \leq c\left(1+\left(\frac{|x|}{\sqrt{t}}\right)^{\tau_{+}(\mu)}+\left(\frac{|y|}{\sqrt{t}}\right)^{\tau_{+}(\mu)}+\left(\frac{|x||y|}{t}\right)^{\tau_{+}(\mu)}\right) t^{-\frac{N}{2}} e^{-c \frac{|x-y|^{2}}{t}},
\end{aligned}
$$

which, together with $\mathcal{G}(x, y)=\int_{0}^{\infty} k_{\mu}(t, x, y) d t$, implies (4.1). 
For $\mu \geq 0$, it follows from Proposition 1.1 in 33 , that

$$
\begin{aligned}
k_{\mu}(t, x, y) & \leq c \min \left\{1,\left(\frac{|x|}{\sqrt{t}}\right)^{\tau_{+}(\mu)}\right\} \min \left\{1,\left(\frac{|y|}{\sqrt{t}}\right)^{\tau_{+}(\mu)}\right\} t^{-\frac{N}{2}} e^{-c \frac{|x-y|^{2}}{t}} \\
& \leq c \min \left\{1,\left(\frac{|x|}{\sqrt{t}}\right)^{\tau_{+}(\mu)},\left(\frac{|y|}{\sqrt{t}}\right)^{\tau_{+}(\mu)},\left(\frac{|x||y|}{t}\right)^{\tau_{+}(\mu)}\right\} t^{-\frac{N}{2}} e^{-c \frac{|x-y|^{2}}{t}}
\end{aligned}
$$

then (4.2) holds.

Remark 4.1 For $\mu_{0}<0$ and $\mu_{0} \leq \mu<0$, it follows from [25] that the heat kernel has the lower bound as

$$
k_{\mu}(t, x, y) \geq c \min \left\{\left(1+\frac{|x|}{\sqrt{t}}\right)^{\tau_{+}(\mu)}\left(1+\frac{|y|}{\sqrt{t}}\right)^{\tau_{+}(\mu)},\left(\frac{d(x) d(x)}{|x||y|}\right)^{\tau_{+}(\mu)}\right\} t^{-\frac{N}{2}} e^{-c \frac{|x-y|^{2}}{t}},
$$

then we have that the Green kernel has lower estimates: for any compact set $K$ in $\Omega$, there exists $c>0$ such that for $x, y \in K \backslash\{0\}$,

$$
\mathcal{G}_{\mu}(x, y) \geq c\left(|x-y|^{2-N}+\frac{|x|^{\tau_{+}(\mu)}}{|x-y|^{N-2+\tau_{+}(\mu)}}+\frac{|y|^{\tau_{+}(\mu)}}{|x-y|^{N-2+\tau_{+}(\mu)}}+\frac{|x|^{\tau_{+}(\mu)}|y|^{\tau_{+}(\mu)}}{|x-y|^{N-2+2 \tau_{+}(\mu)}}\right) .
$$

Proposition 4.1 Let $f$ be a function in $C^{1}(\bar{\Omega} \backslash\{0\})$ satisfying (1.16) and (1.17).

Denote by $\mathbb{G}_{\mu}$ the Green operator defined by

$$
\mathbb{G}_{\mu}[f](x)=\int_{\Omega} \mathcal{G}(x, y) f(y) d y \quad \text { for } \quad f \in L^{1}(\Omega, d \mu) .
$$

Then $\mathbb{G}_{\mu}[f]$ is the classical solution of (1.13) verifying

$$
\lim _{x \rightarrow 0} \mathbb{G}_{\mu}[f](x) \Phi_{\mu}^{-1}(x)=0,
$$

Proof. From the Green's kernel, it is known that $\mathbb{G}_{\mu}[f]$ is the solution of (1.13). We next prove (4.3) and we may assume that $f$ is nonnegative, if not, we just replace $f$ by $|f|$ in the following proof.

Case 1: $N \geq 3$ and $\mu \geq 0$. When $N=2$, there are just some small differences, we omit the proof.

For $x \in \Omega \backslash\{0\}$,

$$
\begin{aligned}
\mathbb{G}_{\mu}[f](x) \Phi_{\mu}^{-1}(x) & \leq c\left(\Phi_{\mu}^{-1}(x) \int_{B_{r_{0}}(0) \backslash B_{\frac{|x|}{2}}(x)} \frac{|y|^{\tau_{+}(\mu)} f(y)}{|x-y|^{N-2+\tau_{+}(\mu)}} d y+\Phi_{\mu}^{-1}(x) \int_{B_{\frac{|x|}{2}}(x)} \frac{f(y)}{|x-y|^{N-2}} d y\right) \\
& =: \quad c\left(\mathbb{E}_{1}(x)+\mathbb{E}_{2}(x)\right),
\end{aligned}
$$

where $r_{0}=\sup _{x, y \in \Omega}|x-y|$ and $f$ is extended to be that $f=0$ in $B_{r_{0}}(0) \backslash \Omega$.

We observe that

$$
\begin{aligned}
\int_{B_{\frac{|x|}{2}}(x)} \frac{f(y)}{|x-y|^{N-2}} d y & \leq \sup _{z \in B_{\frac{3 \mid x x}{2}}(0) \backslash B_{\frac{|x|}{2}}(0)} f(z) \int_{B_{\frac{|x|}{2}}(x)}|x-y|^{2-N} d y \\
& \leq c|x|^{2} \sup _{B_{\frac{3|x|}{2}}(0) \backslash B_{\frac{|x|}{2}}(0)} f(z)
\end{aligned}
$$

and

$$
\mathbb{E}_{2}(x) \leq c \sup _{z \in B_{\frac{3|x|}{2}}(0) \backslash B_{\frac{|x|}{2}}(0)}|z|^{2-\tau_{-}(\mu)} f(z) \rightarrow 0 \quad \text { as } \quad|x| \rightarrow 0
$$


by the assumption (1.17).

For $y \in B_{r_{0}}(0) \backslash B_{\frac{|x|}{2}}(x)$, we have that

$$
|x-y| \geq \frac{1}{8}(|x|+|y|)
$$

and

$$
\begin{aligned}
\int_{B_{r_{0}}(0) \backslash B_{\frac{|x|}{2}}(x)} \frac{|y|^{\tau_{+}(\mu)} f(y)}{|x-y|^{N-2+\tau_{+}(\mu)}} d y & \leq c \int_{B_{r_{0}}(0) \backslash B_{\frac{|x|}{2}}(x)} \frac{|y|^{\tau_{+}(\mu)} f(y)}{(|x|+|y|)^{N-2+\tau_{+}(\mu)}} d y \\
& =c|x|^{2} \int_{B_{\frac{r_{0}}{|x|}(0) \backslash B_{\frac{1}{2}}\left(e_{x}\right)}} \frac{|z|^{\tau_{+}(\mu)} f(|x| z)}{(1+|z|)^{N-2+\tau_{+}(\mu)}} d z .
\end{aligned}
$$

For any $\varepsilon>0$ fixed, there exists $R_{\varepsilon}>0$ such that $1 /\left(1+R_{\varepsilon}\right)^{N-2+\tau_{+}(\mu)}<\varepsilon$, then

$$
\begin{aligned}
\int_{B \frac{r_{0}}{|x|}(0) \backslash B_{R_{\varepsilon}}(0)} \frac{|z|^{\tau_{+}(\mu)} f(|x| z)}{(1+|z|)^{N-2+\tau_{+}(\mu)}} d z & \leq \varepsilon \int_{B \frac{r_{0}(0) \backslash B_{R_{\varepsilon}}(0)}{|x|}}|z|^{\tau_{+}(\mu)} f(|x| z) d z \\
& \leq \varepsilon|x|^{-N-\tau_{+}(\mu)}\|f\|_{L^{1}\left(\Omega, \Gamma_{\mu} d x\right)}
\end{aligned}
$$

and

$$
\begin{aligned}
\int_{B_{R_{\varepsilon}}(0)} \frac{|z|^{\tau_{+}(\mu)} f(|x| z)}{(1+|z|)^{N-2+\tau_{+}(\mu)}} d z & \leq \int_{B_{R_{\varepsilon}}(0)}|z|^{\tau_{+}(\mu)} f(|x| z) d z \\
& \leq|x|^{-N-\tau_{+}(\mu)} \int_{B_{|x| R_{\varepsilon}}(0)}|y|^{\tau_{+}(\mu)} f(y) d y
\end{aligned}
$$

where from (1.16) we have that

$$
\int_{B_{|x| R_{\varepsilon}}(0)}|y|^{\tau_{+}(\mu)} f(y) d y \rightarrow 0 \quad \text { as } \quad|x| \rightarrow 0 .
$$

So $\lim _{|x| \rightarrow 0} \mathbb{E}_{1}(x)=0$. Therefore, along with (4.4) and (4.5),

$$
\lim _{|x| \rightarrow 0} \mathbb{G}_{\mu}[f](x) \Phi_{\mu}^{-1}(x)=0 .
$$

Case 2: $\mu_{0}<0$ and $\mu_{0} \leq \mu<0$. We observe that $N \geq 3$ and

$$
\begin{aligned}
\mathbb{G}_{\mu}[f](x) \leq & c\left(\int_{\Omega} \frac{f(y)}{|x-y|^{N-2}} d y+\int_{\Omega} \frac{|x|^{\tau_{+}(\mu)} f(y)}{|x-y|^{N-2 \tau_{+}(\mu)}} d y\right. \\
& \left.+\int_{\Omega} \frac{|y|^{\tau_{+}(\mu)} f(y)}{|x-y|^{N-2+\tau_{+}(\mu)}} d y+\int_{\Omega} \frac{|x|^{\tau_{+}(\mu)}|y|^{\tau_{+}(\mu)}}{|x-y|^{N-2+2 \tau_{+}(\mu)}} f(y) d y\right) .
\end{aligned}
$$

From (4.5), we have that

$$
\lim _{|x| \rightarrow 0}|x|^{-\tau_{-}(\mu)} \int_{B_{\frac{|x|}{2}}(x)} \frac{f(y)}{|x-y|^{N-2}} d y=0 .
$$

Moreover, we have that

$$
\begin{aligned}
\int_{B_{\frac{|x|}{2}}(x)} \frac{|y|^{\tau_{+}(\mu)} f(y)}{|x-y|^{N-2+\tau_{+}(\mu)}} d y & \leq \sup _{z \in B_{\frac{3|x|}{2}}(0) \backslash B_{\frac{\mid x x}{2}}(0)}|z|^{\tau_{+}(\mu)} f(z) \int_{B_{\frac{|x|}{2}}(x)}|x-y|^{2-N+\tau_{+}(\mu)} d y \\
& \leq c|x|^{2} \sup _{B_{\frac{3|x|}{2}}(0) \backslash B_{\frac{|x|}{2}}(0)} f(z)
\end{aligned}
$$


and then

$$
\lim _{|x| \rightarrow 0}|x|^{-\tau_{-}(\mu)} \int_{B_{\frac{|x|}{2}}(x)} \frac{|y|^{\tau_{+}(\mu)} f(y)}{|x-y|^{N-2+\tau_{+}(\mu)}} d y=0 .
$$

Similarly,

$$
\begin{gathered}
\lim _{|x| \rightarrow 0}|x|^{-\tau_{-}(\mu)} \int_{B_{\frac{|x|}{2}}(x)} \frac{|x|^{\tau_{+}(\mu)} f(y)}{|x-y|^{N-2+\tau_{+}(\mu)}} d y=0 \\
\text { and } \lim _{|x| \rightarrow 0}|x|^{-\tau_{-}(\mu)} \int_{B_{\frac{|x|}{2}}(x)} \frac{|x|^{\tau_{+}(\mu)}|y|^{\tau_{+}(\mu)}}{|x-y|^{N-2+2 \tau_{+}(\mu)}} f(y) d y=0 .
\end{gathered}
$$

For $y \in B_{r_{0}}(0) \backslash B_{\frac{|x|}{2}}(x)$, we have that

$$
|x-y| \geq \frac{1}{8}(|x|+|y|)
$$

and

$$
\begin{aligned}
\int_{B_{r_{0}}(0) \backslash B_{\frac{|x|}{2}}(x)} \frac{f(y)}{|x-y|^{N-2}} d y & \leq c \int_{B_{r_{0}}(0) \backslash B_{\frac{|x|}{2}}(x)} \frac{f(y)}{(|x|+|y|)^{N-2}} d y \\
& =c|x|^{2} \int_{B_{\frac{r_{0}}{|x|}}(0) \backslash B_{\frac{1}{2}}\left(e_{x}\right)} \frac{f(|x| z)}{(1+|z|)^{N-2+\tau_{+}(\mu)}} d z .
\end{aligned}
$$

Fixed $\varepsilon>0$, there exists $R_{\varepsilon}>1$ such that $1 /\left(1+R_{\varepsilon}\right)^{N-2}<\varepsilon$, then for $|z|>R_{\varepsilon}$, we have that

$$
\begin{aligned}
& \left(\frac{1+|z|}{|z|}\right)^{\tau_{+}(\mu)} \leq c \\
& \int_{B_{\frac{r_{0}}{|x|}(0) \backslash B_{R_{\varepsilon}}(0)}} \frac{f(|x| z)}{(1+|z|)^{N-2}} d z \leq c \int_{B_{\frac{r_{0}}{|x|}(0) \backslash B_{R_{\varepsilon}}(0)}} \frac{|z|^{\tau_{+}(\mu)} f(|x| z)}{(1+|z|)^{N-2+\tau_{+}(\mu)}} d z
\end{aligned}
$$

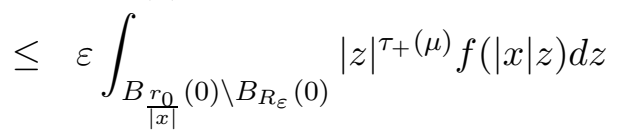

$$
\begin{aligned}
& \leq \varepsilon|x|^{-N-\tau_{+}(\mu)}\|f\|_{L^{1}\left(\Omega, \Gamma_{\mu} d x\right)}
\end{aligned}
$$

and for $|z|<R_{\varepsilon}$, by the fact that $\frac{2-N}{2} \leq \tau_{+}(\mu)<0$,

$$
\begin{aligned}
\int_{B_{R_{\varepsilon}}(0)} \frac{f(|x| z)}{(1+|z|)^{N-2}} d z & \leq \int_{B_{R_{\varepsilon}}(0)}|z|^{\tau_{+}(\mu)} f(|x| z) d z \\
& \leq c|x|^{-N-\tau_{+}(\mu)} \int_{B_{|x| R_{\varepsilon}}(0)}|y|^{\tau_{+}(\mu)} f(y) d y
\end{aligned}
$$

where

$$
\int_{B_{|x| R_{\varepsilon}}(0)}|y|^{\tau_{+}(\mu)} f(y) d y \rightarrow 0 \quad \text { as } \quad|x| \rightarrow 0 .
$$

So

$$
\lim _{|x| \rightarrow 0} \Phi_{\mu}^{-1}(x) \int_{B_{r_{0}}(0) \backslash B_{\frac{|x|}{2}}(x)} \frac{f(y)}{|x-y|^{N-2}} d y=0,
$$


then we have that

$$
\lim _{|x| \rightarrow 0}\left(\int_{\Omega} \frac{f(y)}{|x-y|^{N-2}} d y\right) \Phi_{\mu}^{-1}(x)=0 .
$$

Similar we can prove

$$
\begin{aligned}
& \lim _{|x| \rightarrow 0}\left(\int_{\Omega} \frac{|x|^{\tau_{+}(\mu)} f(y)}{|x-y|^{N-2+\tau_{+}(\mu)}} d y\right) \Phi_{\mu}^{-1}(x)=0, \\
& \lim _{|x| \rightarrow 0}\left(\int_{\Omega} \frac{|y|^{\tau_{+}(\mu)} f(y)}{|x-y|^{N-2+\tau_{+}(\mu)}} d y\right) \Phi_{\mu}^{-1}(x)=0
\end{aligned}
$$

and

$$
\lim _{|x| \rightarrow 0}\left(\int_{\Omega} \frac{|x|^{\tau_{+}(\mu)}|y|^{\tau_{+}(\mu)} f(y)}{|x-y|^{N-2+2 \tau_{+}(\mu)}} d y\right) \Phi_{\mu}^{-1}(x)=0 .
$$

Thus (4.3) holds.

Remark 4.2 We remark that for $\tau>\tau_{-}(\mu)$, if

$$
\limsup _{x \rightarrow 0} f(x)|x|^{-\tau+2}<+\infty,
$$

then $f$ verifies (1.16) and (1.17). Furthermore, when $\mu>\mu_{0}$ and $f \in C_{\text {loc }}^{\gamma}(\bar{\Omega} \backslash\{0\})$ verifies 4.6], there exists $c>0$ such that

$$
\left|\mathbb{G}_{\mu}[f](x)\right| \leq c|x|^{\min \left\{\tau, \tau_{+}(\mu)\right\}} .
$$

\subsection{Existence and nonexistence}

This subsection is devoted to build the distributional identity for the nonhomogeneous problem (1.13).

Lemma 4.2 Assume that $f$ is a function in $C_{l o c}^{\gamma}(\bar{\Omega} \backslash\{0\})$ satisfying (1.16). Then for $\forall k \in \mathbb{R}$, problem (1.14) admits a unique weak solution $u_{k}$, has a unique solution $u_{k}$, which is a classical solution of problem (1.13).

Furthermore, if (1.17) holds true, then $\lim _{x \rightarrow 0} u_{k}(x) \Phi_{\mu}^{-1}(x)=k$.

Proof. Let $f_{n}=f \eta_{n}$, where $\eta_{n}(r)=1-\eta_{0}(n r)$ for $r \geq 0$. We see that $f_{n}$ is bounded. Let $v_{n}$ and $v_{n}^{+}$be the solution of (2.4) replaced $f$ by $f_{n}$ and $\left|f_{n}\right|$ respectively, then

$$
\left|v_{n}\right| \leq v_{n}^{+}
$$

and for any $\xi \in C_{0}^{1.1}(\Omega)$,

$$
\int_{\Omega} v_{n} \mathcal{L}_{\mu}^{*}(\xi) d \mu=\int_{\Omega} f_{n} \xi d \mu, \quad \int_{\Omega} v_{n}^{+} \mathcal{L}_{\mu}^{*}(\xi) d \mu=\int_{\Omega}\left|f_{n}\right| \xi d \mu
$$

Take $\xi=\xi_{0}$ as before, we have that $v_{n}^{+}$is uniformly bounded in $L^{1}(\Omega, d \mu)$ by (1.16) , so is $v_{n}$.

Claim 1: $v_{n}^{+}$is uniform bounded in $L^{1}\left(\Omega,|x|^{-1} d \mu\right)$. For $\sigma>0$, let $\xi_{\sigma}$ be defined in (3.8), then using the expression and the estimate of $\mathcal{L}_{\mu}^{*}\left(\xi_{\sigma}\right)(x)$ in $B_{\frac{1}{n_{0}}}(0)$ given by (3.9) and (3.10) respectively, we have that

$$
\begin{aligned}
\left(N-1+\tau_{+}(\mu)\right) \int_{B_{\frac{1}{n_{0}}}(0) \backslash B_{\sigma}(0)} v_{n}^{+}|x|^{-1} d \mu & \leq \int_{B_{\frac{1}{n_{0}}}(0)} v_{n}^{+} \mathcal{L}_{\mu}^{*}\left(\xi_{\sigma}\right) d \mu \\
& \leq \int_{\Omega}|f| \xi_{\sigma} d \mu+c \int_{\Omega \backslash B_{\frac{1}{n_{0}}}(0)} v_{n}^{+} d \mu \\
& \leq c \int_{\Omega}|f| \rho d \mu
\end{aligned}
$$


Passing to the limit as $\sigma \rightarrow 0$, we have that

$$
\int_{B_{\frac{1}{n_{0}}}(0)} v_{n}^{+}|x|^{-1} d \mu \leq c^{\prime} \int_{\Omega}|f| \rho d \mu,
$$

which, together with the fact that

$$
\int_{\Omega \backslash B \frac{1}{n_{0}}(0)} v_{n}^{+}|x|^{-1} d \mu \leq c \int_{\Omega}|f| \rho d \mu,
$$

implies that

$$
\int_{\Omega} v_{n}^{+}|x|^{-1} d \mu \leq c^{\prime} \int_{\Omega}|f| \rho d \mu
$$

that is, $v_{n}^{+}$is uniform bounded in $L^{1}\left(\Omega,|x|^{-1} d \mu\right)$.

Moreover, $\left\{v_{n}^{+}\right\}$is increasing, and then there exists $v_{+}$such that

$$
v_{n}^{+} \rightarrow v_{+} \text {a.e. in } \Omega \text { and in } L^{1}\left(\Omega,|x|^{-1} d \mu\right) .
$$

Then we have that

$$
\int_{\Omega} v_{+} \mathcal{L}_{\mu}^{*}(\xi) d \mu=\int_{\Omega}|f| \xi d \mu, \quad \forall \xi \in C_{0}^{1.1}(\Omega)
$$

Since $f \in C^{\gamma}(\bar{\Omega} \backslash\{0\})$, then from Lemma 4.10 in [18], we have that $v \in C^{1}(\Omega \backslash\{0\})$ and then from (4.7), up to subsequence, there exists $v_{f}$ such that

$$
v_{n} \rightarrow v_{f} \quad \text { in } C^{1}(\Omega \backslash\{0\}) \text { and in } L^{1}(\Omega, d \mu)
$$

and

$$
\int_{\Omega} v_{f} \mathcal{L}_{\mu}^{*}(\xi) d \mu=\int_{\Omega} f \xi d \mu, \quad \forall \xi \in C_{0}^{1.1}(\Omega),
$$

thus, $u_{k, f}=k G_{\mu}+v_{f}$ is a weak solution of (1.14) and the uniqueness follows by the Kato's inequality Proposition 2.1.

Claim 2: $v_{f}$ is a classical solution of (1.13) subject to $\lim _{x \rightarrow 0} u(x) \Phi_{\mu}^{-1}(x)=0$. From Corollary 2.8 in [45] with $L^{*}=\mathcal{L}_{\mu}^{*}$, which is strictly elliptic in $\Omega \backslash B_{r}(0)$, we have that for $q<\frac{N}{N-1}$,

$$
\begin{aligned}
\left\|v_{n} \Gamma_{\mu}\right\|_{W^{1, q}\left(\Omega_{2 r}\right)} & \leq c\left\|f \Gamma_{\mu}\right\|_{L^{1}\left(\Omega \backslash B_{r}(0)\right)}+c\left\|v_{n} \Gamma_{\mu}\right\|_{L^{1}\left(\Omega \backslash B_{r}(0)\right)} \\
& \leq c\|f\|_{L^{1}(\Omega, d \mu)}
\end{aligned}
$$

where $\Omega_{2 r}=\left\{x \in \Omega \backslash B_{2 r}(0): \rho(x)>2 r\right\}$. We see that

$$
-\Delta v_{n}=-\frac{\mu}{|x|^{2}} v_{n}+f .
$$

For any compact set $K$ in $\Omega$ away from the origin, it is standard to improve the regularity $v_{n}$

$$
\left\|v_{n}\right\|_{C^{3}(K)} \leq c\left[\|f\|_{L^{1}(\Omega, d \mu)}+\|f\|_{C^{1}(K)}\right]
$$

where $c>0$ is independent of $n$. Then $v_{f}$ is a classical solution of (1.13) by the stability theorem, so is $k G_{\mu}+v_{f}$.

We observe that

$$
v_{n}=\mathbb{G}_{\mu}\left[f_{n}\right] \text { and } v_{f}=\lim _{n \rightarrow+\infty} \mathbb{G}_{\mu}\left[f_{n}\right]=\mathbb{G}_{\mu}[f],
$$

then it deduces from Proposition 4.1 if (1.17) holds true, that $\lim _{x \rightarrow 0} v_{f}(x) \Phi_{\mu}^{-1}(x)=0$. We complete the proof.

Lemma 4.3 Assume that $f$ is a nonnegative function in $C_{\text {loc }}^{\gamma}(\bar{\Omega} \backslash\{0\})$ satisfying (1.19)). Then problem (1.13) has no nonnegative solution. 
Proof. By contradiction, we assume that problem (1.13) has a nonnegative solution of $u_{f}$. Let $\left\{r_{n}\right\}_{n}$ be a sequence of strictly decreasing positive numbers converging to zero. From (1.19) and the fact $f \in C^{\gamma}(\bar{\Omega} \backslash\{0\})$, for any $r_{n}$, we have that

$$
\lim _{r \rightarrow 0^{+}} \int_{B_{r_{n}}(0) \backslash B_{r}(0)} f(x) d \mu=+\infty,
$$

then there exists $R_{n} \in\left(0, r_{n}\right)$ such that

$$
\int_{B_{r_{n}}(0) \backslash B_{R_{n}}(0)} f d \mu=n,
$$

The case of $\mu \geq 0$. Let $\delta_{n}=\frac{1}{n} \Gamma_{\mu} f \chi_{B_{r_{n}}(0) \backslash B_{R_{n}}(0)}$, then the problem

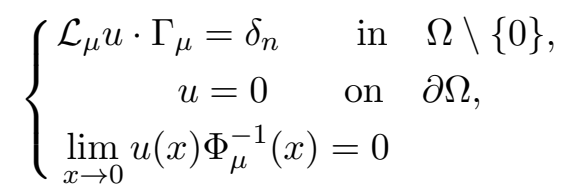

has a unique positive solution $w_{n}$ satisfying (in the usual sense)

$$
\int_{\Omega} w_{n} \mathcal{L}_{\mu}\left(\Gamma_{\mu} \xi\right) d x=\int_{\Omega} \delta_{n} \xi d x, \quad \forall \xi \in C_{0}^{1.1}(\Omega) .
$$

For any $\xi \in C_{0}^{1.1}(\Omega)$, we have that

$$
\int_{\Omega} w_{n} \mathcal{L}_{\mu}^{*}(\xi) d \mu=\int_{\Omega} \delta_{n} \xi d x \rightarrow \xi(0) \quad \text { as } \quad n \rightarrow+\infty .
$$

Therefore, by Lemma 3.1 for any compact set $\mathcal{K} \subset \Omega \backslash\{0\}$

$$
\left\|w_{n}-G_{\mu}\right\|_{C^{1}(\mathcal{K})} \rightarrow 0 \quad \text { as } \quad n \rightarrow+\infty .
$$

So we fixed a point $x_{0} \in \Omega \backslash\{0\}$, let $r_{0}=\frac{\min \left\{\left|x_{0}\right|, \rho\left(x_{0}\right)\right\}}{2}$ and $\mathcal{K}=\overline{B_{r_{0}}\left(x_{0}\right)}$, then there exists $n_{0}>0$ such that for $n \geq n_{0}$,

$$
w_{n} \geq \frac{1}{2} G_{\mu} \quad \text { in } \quad \mathcal{K} .
$$

Let $u_{n}$ be the solution (in the usual sense) of

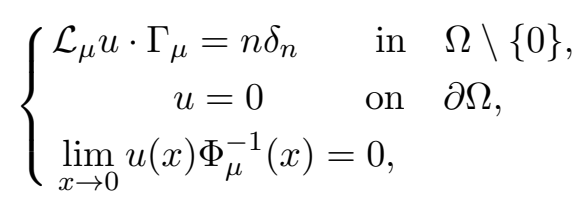

then we have that

$$
u_{n} \geq n w_{n} \quad \text { in } \quad \Omega \text {. }
$$

Together with (4.9), we derive that

$$
u_{n} \geq \frac{n}{2} G_{\mu} \quad \text { in } \quad \mathcal{K} .
$$

Then by Comparison Principle, we have that

$$
u_{f}\left(x_{0}\right) \geq u_{n}\left(x_{0}\right) \rightarrow+\infty \quad \text { as } n \rightarrow+\infty,
$$

which contradicts that $u_{f}$ is classical solution of (1.13). 
The case of $\mu_{0} \leq \mu<0$. Let $w_{n}$ be the solution of

$$
\left\{\begin{aligned}
\mathcal{L}_{\mu} u(x)=f \chi_{B_{r_{n}}(0) \backslash B_{R_{n}}(0)} & \forall x \in \Omega \backslash\{0\}, \\
u(x)=0 & \forall x \in \partial \Omega, \\
\lim _{x \rightarrow 0} u(x) \Phi_{\mu}^{-1}(x)=0, &
\end{aligned}\right.
$$

then it follows by comparison principle

$$
u \geq w_{n}(x) \geq \int_{B_{r_{n}}(0) \backslash B_{R_{n}}(0)} \mathcal{G}_{\mu}(x, y) f(y) d y .
$$

It follows from Remark 4.1 that for $x, y \in B_{3}(0) \backslash\{0\}, x \neq y$,

$$
\mathcal{G}_{\mu}(x, y) \geq c\left(|x-y|^{2-N}+\frac{|x|^{\tau_{+}(\mu)}}{|x-y|^{N-2+\tau_{+}(\mu)}}+\frac{|y|^{\tau_{+}(\mu)}}{|x-y|^{N-2+\tau_{+}(\mu)}}+\frac{|x|^{\tau_{+}(\mu)}|y|^{\tau_{+}(\mu)}}{|x-y|^{N-2+2 \tau_{+}(\mu)}}\right) .
$$

For $x_{0} \in \mathbb{R}^{N}$ with $\left|x_{0}\right|=2$ fixed, it deduce that

$$
\begin{aligned}
u\left(x_{0}\right) \geq w_{n}\left(x_{0}\right) & \geq c 2^{\tau_{+}(\mu)} \int_{B_{2}(0)} \frac{|y|^{\tau_{+}(\mu)}}{\left|x_{0}-y\right|^{N-2+2 \tau_{+}(\mu)}} f(y) d y \\
& \geq c \int_{B_{r_{n}}(0) \backslash B_{R_{n}}(0)} f d \mu=c n \rightarrow+\infty \text { as } n \rightarrow+\infty,
\end{aligned}
$$

which is impossible.

\subsection{Classification}

In this subsection, we are devoted to classify the isolated singular solutions of (1.13) in the distributional sense. When $\mu=0$, the related classification of isolated singularities was studied in [9].

Proposition 4.2 Assume that $f$ is a function in $C_{l o c}^{\gamma}(\bar{\Omega} \backslash\{0\})$ satisfying (1.16) and $u$ is a non-

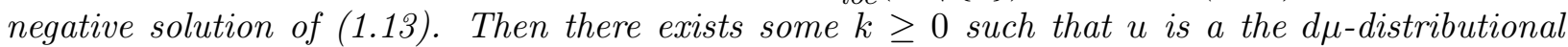
solution of (1.15).

Proof. Let

$$
\bar{u}(r)=\left|\mathcal{S}^{N}\right|^{-1} r^{1-N} \int_{\partial B_{r}(0)} u(x) d \omega(x) .
$$

For $r \in\left(0, r_{0}\right)$, we have that

$$
-\bar{u}^{\prime \prime}(r)-\frac{N-1}{r} \bar{u}^{\prime}(r)+\frac{\mu}{r^{2}} \bar{u}(r) \geq \bar{f}(r),
$$

where

$$
\bar{f}(r)=r^{1-N} \int_{\partial B_{r}(0)} f(x) d \omega(x) .
$$

Denote

$$
\bar{u}(r)=r^{\tau_{+}(\mu)} v(r)
$$

then

$$
-v^{\prime \prime}(r)-\frac{N+2 \tau_{+}(\mu)-1}{r} v^{\prime}(r) \geq r^{-\tau_{+}(\mu)} \bar{f},
$$

where $N+2 \tau_{+}(\mu)$ plays the dimensional role. By (1.16), we have that

$$
\int_{0}^{r_{0}} r^{-\tau_{+}(\mu)} \bar{f}(r) r^{N+2 \tau_{+}(\mu)-1} d r=\int_{B_{r_{0}}(0)}|x|^{-\tau_{+}(\mu)}|f(x)| d x<+\infty,
$$


where $r_{0}>0$ such that $B_{r_{0}}(0) \subset \Omega$. Following the step 1 in the proof of Theorem 1.1 in [9], there exists $c>0$ such that

$$
v(r) \leq\left\{\begin{array}{lll}
c|x|^{2-\left(N+2 \tau_{+}(\mu)\right)} & \text { if } & N+2 \tau_{+}(\mu) \geq 3, \\
-c \ln |x| & \text { if } & N+2 \tau_{+}(\mu)=2,
\end{array}\right.
$$

that is, $\bar{u} \leq c \Phi_{\mu}$. So for $\xi \in C_{c}^{\infty}(\Omega)$, it is well-defined that

$$
\begin{aligned}
\left|\int_{\Omega} u \mathcal{L}_{\mu}^{*}(\xi) d \mu\right| & \leq\|\xi\|_{C^{2}} \int_{B_{r_{0}}(0)} u(x)|x|^{-1} \Gamma_{\mu}(x) d x+c \\
& \leq c\|\xi\|_{C^{2}} \int_{0}^{r_{0}} \bar{u}(r) r^{-1+\tau_{+}(\mu)} r^{N-1} d r+c \\
& \leq c\|\xi\|_{C^{2}} \int_{0}^{r_{0}} r^{\tau_{-}(\mu)+\tau_{+}(\mu)+N-2} d r+c \\
& <+\infty .
\end{aligned}
$$

We observe that for $\xi \in C_{c}^{\infty}(\Omega \backslash\{0\})$, it follows by Divergence theorem that

$$
\int_{\Omega} u \mathcal{L}_{\mu}^{*}(\xi) \Gamma_{\mu} d x-\int_{\Omega} f \xi \Gamma_{\mu} d x=0
$$

By Schwartz Theorem ([40, Theorem XXXV]), there exists a multiple index $p$,

$$
u \Gamma_{\mu} \mathcal{L}_{\mu}^{*}-f \Gamma_{\mu}=\sum_{|a|=0}^{p} k_{a} D^{a} \delta_{0},
$$

i.e. for any $\xi \in C_{c}^{\infty}(\Omega)$

$$
\int_{\Omega} u\left(\mathcal{L}_{\mu}^{*} \xi-f \xi\right) d \mu=\sum_{|a|=0}^{|p|} k_{a} D^{a} \xi(0) .
$$

We are left to show that $k_{a}=0$ for $|a| \geq 1$. For multiple index $\bar{a} \neq 0$, taking $\xi_{\bar{a}}(x)=x_{i}^{\bar{a}_{i}} \eta_{n_{0}}$ and denoting $\xi_{\bar{a}, \varepsilon}(x)=\xi_{\bar{a}}\left(\frac{x}{\varepsilon}\right)$, we have that $\xi_{\bar{a}} \in C_{c}^{\infty}(\Omega)$ and then for $\varepsilon \in\left(0, \frac{1}{2}\right)$,

$$
\mathcal{L}_{\mu}^{*} \xi_{\bar{a}, \varepsilon}(x)=\frac{1}{\varepsilon^{2}}(-\Delta) \xi_{\bar{a}}\left(\frac{x}{\varepsilon}\right)-\frac{2}{\varepsilon} \frac{x}{|x|^{2}} \cdot \nabla \xi_{\bar{a}}\left(\frac{x}{\varepsilon}\right),
$$

and on the one side,

$$
\begin{aligned}
\left|\int_{\Omega} u \mathcal{L}_{\mu}^{*}\left(\xi_{\bar{a}, \varepsilon}\right) d \mu\right| & =\left|\frac{1}{\varepsilon^{2}} \int_{B_{2 \varepsilon}(0)} u_{\mu} \Gamma_{\mu}(-\Delta) \xi_{\bar{a}}\left(\frac{x}{\varepsilon}\right) d x-\frac{1}{\varepsilon} \int_{B_{2 \varepsilon}(0)} u_{\mu} \Gamma_{\mu} \frac{x}{|x|^{2}} \cdot \nabla \xi_{\bar{a}}\left(\frac{x}{\varepsilon}\right)\right| \\
& \leq \frac{1}{\varepsilon^{2}} \int_{B_{2 \varepsilon}(0)} u_{\mu} \Gamma_{\mu} d x+\frac{1}{\varepsilon} \int_{B_{2 \varepsilon}(0)} \frac{u_{\mu} \Gamma_{\mu}}{|x|} d x \\
& \leq \begin{cases}c & \text { if } \quad N \geq 3, \\
-c \ln \varepsilon & \text { if } \quad N=2,\end{cases}
\end{aligned}
$$

where $c$ is independent of $\varepsilon$. Moreover, we have that

$$
\left|\int_{\Omega} u_{\mu} \xi_{\bar{a}, \varepsilon} d \mu\right| \leq\left\|\xi_{\bar{a}}\right\|_{L^{\infty}} \int_{B_{2 \varepsilon}(0)} f_{\mu} \Gamma_{\mu} d x \rightarrow 0 \quad \text { as } \varepsilon \rightarrow 0^{+} .
$$

On the other side,

$$
\sum_{|a|=0}^{|p|} k_{a} D^{a} \xi_{\bar{a}, \varepsilon}(0)=\frac{k_{\bar{a}}}{\varepsilon^{|\bar{a}|}}|\bar{a} !|
$$


where

$$
|\bar{a}|=\sum \bar{a}_{i} \quad \text { and } \quad \bar{a} !=\prod_{i=1}^{N}\left(\bar{a}_{i}\right) ! \geq 1 .
$$

So if $k_{\bar{a}} \neq 0$, we have that

$$
\left|\sum_{|a|=0}^{|p|} k_{a} D^{a} \xi_{\bar{a}, \varepsilon}(0)\right| \rightarrow+\infty \quad \text { as } \varepsilon \rightarrow 0^{+},
$$

that is, the right hand of (4.10) with $\xi=\xi_{\bar{a}, \varepsilon}$ blows up with the rate $\varepsilon^{-|\bar{a}|}$, while the left hand of (4.10) keeps bounded for $N \geq 3$ or blows up in $N=2$, but controlled by $-\ln \varepsilon$ as $\varepsilon \rightarrow 0^{+}$. This is a contradiction and so $k_{a}=0$ for $|a| \geq 1$.

Therefore, we have that

$$
\int_{\Omega}\left(u_{\mu} \mathcal{L}_{\mu}^{*} \xi-f \xi\right) d \mu=k_{0} \xi(0), \quad \forall \xi \in C_{c}^{\infty}(\Omega) .
$$

For $\xi \in C_{0}^{1.1}(\Omega)$, take a sequence of functions in $C_{c}^{\infty}(\Omega)$ converging to $\xi$, then the identity (4.11) holds for any $\xi \in C_{0}^{1.1}(\Omega)$.

Proof of Theorem 1.3. The part (i) follows by Lemma 4.2 and Proposition 4.1, the part (ii) does by Proposition 4.2. Lemma 4.3 implies the nonexistence in part (iii).

\section{Generalization and Application}

\section{$5.1 d \mu$-distributional solution}

In this subsection, we prove existence of $d \mu$-distributional solution of (1.14) with a general nonhomogeneous term.

Proposition 5.1 Assume that $\Omega$ is a smooth bounded domain containing the origin in $\mathbb{R}^{N}, \rho(x):=$ $\operatorname{dist}(x, \partial \Omega)$ and $f$ is a measurable function satisfying

$$
\int_{\Omega}|f| \rho d \mu<+\infty
$$

Then for any $k \in \mathbb{R}$, problem (1.14) admits a unique d $\mu$-distributional solution.

Proof. By the linearity of $\mathcal{L}_{\mu}$, we find out the solutions $u_{+}$and $u_{-}$of (1.14) with the nonhomogeneous term $f_{+}+k_{+} \delta_{0}$ and $f_{-}+k_{-} \delta_{0}$, respectively, where $a_{ \pm}=\max \{ \pm a, 0\}$. Then $u=u_{+}-u_{-}$is the solution of (1.14). So we assume now that $f$ is nonnegative and $k \geq 0$. We observe that there exists a increasing nonnegative sequence $f_{n} \in C^{1}(\bar{\Omega})$ such that

$$
f_{n} \rightarrow f \quad \text { as } \quad n \rightarrow \infty \quad \text { in } L^{1}(\Omega, \rho d \mu) .
$$

From Theorem 1.3, we have that problem (1.13), subject to $\lim _{x \rightarrow 0} u(x) \Phi_{\mu}^{-1}(x)=k$, has a unique solution of $u_{n}$ satisfying

$$
\int_{\Omega} u_{n} \mathcal{L}_{\mu}^{*}(\xi) d \mu=\int_{\Omega} f_{n} \xi d \mu+c_{\mu} k \xi(0) .
$$

By the Comparison Principle, we have that

$$
0 \leq u_{n} \leq u_{n+1} \quad \text { in } \quad \Omega \backslash\{0\} .
$$

Now take $\xi=\xi_{0}$, the solution of (2.2), we get that

$$
\left\|u_{n}\right\|_{L^{1}(\Omega, d \mu)} \leq \int_{\Omega}|f| \rho d \mu+c k,
$$


where $c>0$ is independent of $n$. By Claim 1 in the proof of Lemma 4.2, we have that

$$
\int_{\Omega} u_{n}|x|^{-1} d \mu \leq c \int_{\Omega}|f| \rho d \mu .
$$

Since $u_{n}$ is increasing and uniformly bounded in $L^{1}\left(\Omega,|x|^{-1} d \mu\right)$, by Lebesgue's monotone convergence theorem, we have that $u(x):=\lim _{n \rightarrow+\infty} u_{n}$ is in $L^{1}\left(\Omega,|x|^{-1} d \mu\right)$ and satisfies

$$
\int_{\Omega} u \mathcal{L}_{\mu}^{*}(\xi) d \mu=\int_{\Omega} f_{n} \xi d \mu+c_{\mu} k \xi(0), \quad \forall \xi \in C_{0}^{1.1}(\Omega) .
$$

The uniqueness follows by Kato's inequality directly. Indeed, let $u_{1}, u_{2}$ be two solutions of (1.14), then $w:=u_{1}-u_{2}$ is a $d \mu$-distributional solution of (2.9) with $f=0$, taking $\xi=\xi_{0}$ in (2.10), $\xi_{0}$ being the solution of (2.2), it follows that $\int_{\Omega}|w| d x=0$, then $u_{1}=u_{2}$. This ends the proof.

\subsection{Nonexistence on $\mu<\mu_{0}$}

Although we have supposed $\mu \geq \mu_{0}$ throughout this paper at the beginning, we can still deal the case of $\mu<\mu_{0}$ and obtain an Liouville Theorem in this case.

Proposition 5.2 Assume that $\mu<\mu_{0}$ and $f$ is a measurable nonnegative function, then problem (1.13) has no nontrivial nonnegative solutions.

Proof. By contradiction, we assume that $u_{0}$ is a nontrivial nonnegative solution of (1.13). Then there exist $x_{0} \in \Omega \backslash\{0\}, r \in\left(0,\left|x_{0}\right|\right)$ and $\epsilon_{0}>0$ such that $B_{2 r_{0}}\left(x_{0}\right) \subset \Omega \backslash\{0\}$ and

$$
u_{0} \geq \epsilon_{0} \quad \text { in } \quad B_{r}\left(x_{0}\right) .
$$

We observe that

$$
\mathcal{L}_{\mu_{0}} u_{0}=\left(\mu_{0}-\mu\right) \frac{u_{0}}{|x|^{2}}+f \geq\left(\mu_{0}-\mu\right) \epsilon_{0} \frac{\chi_{B_{r_{0}}\left(x_{0}\right)}}{|x|^{2}},
$$

where $\mu_{0}-\mu>0, \chi_{B_{r_{0}}\left(x_{0}\right)}=1$ in $B_{r_{0}}\left(x_{0}\right)$ and $\chi_{B_{r_{0}}\left(x_{0}\right)}=0$ otherwise.

When $N \geq 3$, from Remark 4.1 with $K=\overline{B_{r_{0}}\left(x_{0}\right) \cup B_{r_{0}}(0)}$, for $x \in B_{r_{0}}(0) \backslash\{0\}$,

$$
\begin{aligned}
u_{0}(x) & \geq\left(\mu_{0}-\mu\right) \epsilon_{0} \mathbb{G}_{\mu_{0}}\left[\chi_{B_{r_{0}}\left(x_{0}\right)}\right] \\
& \geq\left(\mu_{0}-\mu\right) \epsilon_{0} c|x|^{-\frac{N-2}{2}} \int_{B_{r_{0}}\left(x_{0}\right)} \frac{1}{|x-y|^{\frac{N-2}{2}}} d y \\
& \geq c_{0}|x|^{-\frac{N-2}{2}}
\end{aligned}
$$

where $c_{0}=\left(\mu_{0}-\mu\right) \epsilon_{0} c \min _{x \in \Omega} \int_{B_{r_{0}}\left(x_{0}\right)} \frac{1}{|x-y|^{\frac{N-2}{2}}} d y>0$. Go back to (5.2), we see that

$$
\begin{aligned}
\int_{\Omega \backslash B_{r}(0)}\left[\left(\mu_{0}-\mu\right) \frac{u_{0}}{|x|^{2}}+f\right] d \mu & \geq c_{0} \int_{B_{r_{0}}(0) \backslash B_{r}(0)}|x|^{-N} d x \\
& \rightarrow+\infty \quad \text { as } \quad r \rightarrow 0^{+} .
\end{aligned}
$$

Apply Theorem [1.3( (iii), we obtain that

$$
\left\{\begin{aligned}
\mathcal{L}_{\mu} u & =\left(\mu_{0}-\mu\right) \frac{u_{0}}{|x|^{2}}+f \quad \text { in } \quad \Omega \backslash\{0\}, \\
u & =0 \quad \text { on } \partial \Omega
\end{aligned}\right.
$$

has no nonnegative solution, which contradicts that $u_{0}$ is a nonnegative solution (5.3). So we conclude that problem (1.13) has no nontrivial nonnegative solutions. 
When $N=2$ and $\mu_{0}=0$ we have that for any compact set $K$ in $\Omega$, we have that $\mathcal{G}_{0}(x, y) \geq c^{\prime}$ for $x, y \in K, x \neq y$,

$$
\begin{aligned}
u_{0}(x) & \geq\left(\mu_{0}-\mu\right) \epsilon_{0} \mathbb{G}_{\mu_{0}}\left[\chi_{B_{r_{0}}\left(x_{0}\right)}\right] \\
& \geq\left(\mu_{0}-\mu\right) \epsilon_{0} c \int_{B_{r_{0}}\left(x_{0}\right)}(-\ln |x-y|) d y \\
& \geq\left(\mu_{0}-\mu\right) \epsilon_{0} c\left|B_{r_{0}}\left(x_{0}\right)\right| c^{\prime} .
\end{aligned}
$$

Then a contradiction could be obtained as the case of $N \geq 3$.

\subsection{Nonexistence of the principle eigenvalue}

In this subsection, our aim is to study the nonexistence of principle eigenvalue of the problem

$$
\left\{\begin{array}{l}
-\Delta u=\lambda V u \quad \text { in } \quad \mathcal{O}, \\
u \in H_{0}^{1}(\mathcal{O}),
\end{array}\right.
$$

where $\mathcal{O}$ is an smooth open set containing $\{0\}$ in $\mathbb{R}^{N}$ and $V$ is a measurable function.

It was proved in [42] (Theorem 2.5) that problem (5.4) admits a simple principle eigenvalue under the following hypotheses $\left(V_{1}\right)$ and $\left(V_{2}\right)$ :

$\left(V_{1}\right) V \in L_{l o c}^{p}(\mathcal{O} \backslash\{0\})$ with $p>\frac{N}{2} ;$

$\left(V_{2}\right)$ Let

$$
V^{+}:=\max \{0, V\} \neq 0, \quad V^{+}=V_{1}+V_{2},
$$

where $V_{1} \in L^{\frac{N}{2}}(\mathcal{O})$ and for any $y \in \overline{\mathcal{O}}$,

$$
\lim _{x \in \mathcal{O}, x \rightarrow y}|x-y|^{2} V_{2}(x)=0, \quad \lim _{x \in \mathcal{O}, x \rightarrow \infty}|x|^{2} V_{2}(x)=0 \text { (if } \mathcal{O} \text { is unbounded). }
$$

More references on the principle eigenvalue with indefinite potential also could be seen in [13, 15, 41, 46].

Our aim here is to the nonexistence of the principle eigenvalue if $V$ is the Hardy-Leray potential in bounded domain. We will see that the assumptions on $V$ above seem to be "optimal" in some sense. More precisely, our result can be stated as follows:

Theorem 5.1 Assume that $\mathcal{O}=\Omega$ is a bounded $C^{2}$ domain containing the origin, the potential $V \in C_{l o c}^{\gamma}(\bar{\Omega} \backslash\{0\})$ verifies

$$
V(x) \geq \frac{a_{0}}{|x|^{2}}, \quad \forall x \in \Omega \backslash\{0\}
$$

for some constants $\gamma \in(0,1)$ and $a_{0}>0$. Then problem (5.4) has no principle eigenvalue.

Proof. By contradiction, we assume that problem (5.4) has a principle eigenvalue $\lambda_{1}$, and denote by $\varphi_{1}$ the nonnegative corresponding eigenfunction.

Since $V$ is nonnegative and $V \in C^{\gamma}(\bar{\Omega} \backslash\{0\})$, it is standard to prove that $\lambda_{1}>0$ and $\varphi_{1} \in H_{0}^{1}(\Omega)$ is a nonnegative classical solution of

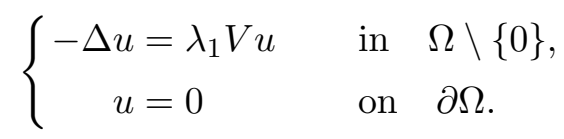

So $\varphi_{1}$ a super solution of

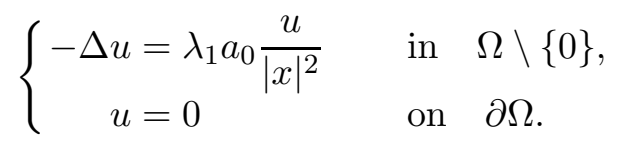


Let $\psi_{1}$ be the first positive eigenfunction of $-\Delta u=\lambda u$ in $H_{0}^{1}\left(\Omega \backslash B_{r}(0)\right)$ with $r>0$ small. Then there exists $\epsilon \in(0,1)$ such that $\epsilon \psi_{1}$ is a sub solution of (5.7). By the super and sub solutions methods, problem (5.7) has a nonnegative nontrivial solution $v_{1} \in H_{0}^{1}(\Omega)$.

In the case that $\lambda_{1} a_{0} \in\left(0,-\mu_{0}\right]$, it follows by Theorem 1.3 (iii) with $f=0$, we have that $v_{1}=k G_{-\lambda_{1} a_{0}}$ for some $k \geq 0$. If $k=0, v_{0}=0$ is trivial, which is impossible. If $k>0$, we know that $G_{-\lambda_{1} a_{0}} \notin H_{0}^{1}(\Omega)$, which contradicts $v_{1} \in H_{0}^{1}(\Omega)$.

In the case that $\lambda_{1} a_{0}>-\mu_{0}$, it follows by Proposition 5.2 that there is no nonnegative nontrivial solution. So we obtain a contradictions. This ends the proof.

Acknowledgements: The authors would like to thank Prof. Brezis for useful discussing. H. Chen is supported by NNSF of China, No:11726614, 11661045, by SRF for ROCS, SEM and by the Jiangxi Provincial Natural Science Foundation, No: 20161ACB20007. A. Quaas is partially supported by Fondecyt Grant No. 1151180 Programa Basal, CMM, U. de Chile and Millennium Nucleus Center for Analysis of PDE NC130017. F. Zhou is partially supported by NNSF of China, No:11271133, 11431005 and Shanghai Key Laboratory of PMMP.

\section{References}

[1] O. Adimurthi, N. Chaudhuri and M. Ramaswamy, An improved Hardy-Sobolev inequality and its application, Proc. Amer. Math. Soc., 130, (2002), 489-505.

[2] P. Baras and J. Goldstein, The heat equation with a singular potential, Trans. Amer. Math. Soc. 284(1), (1984), 121-139.

[3] P. Baras and J. Goldstein, Remarks on the inverse square potential in quantum mechanics, Differential equations (Birmingham, Ala.), (1984), 31-35.

[4] C. Bandle, M. Marcus and V. Moroz, Boundary singularities of solutions of semilinear elliptic equations in the half-space with a Hardy potential, arXiv:1604.08830.

[5] Ph. Bénilan, H. Brezis and M. Crandall, A semilinear elliptic equation in $L^{1}\left(\mathbb{R}^{N}\right)$, Ann. Sc. Norm. Sup. Pisa Cl. Sci., 2, (1975), 523-555.

[6] M. Bidaut-Véron, Singularities of solutions of a class of quasilinear equations in divergence form, Nonlinear Diffusion Equations and Their Equilibrium States, 3, (1992), 129-144.

[7] L. Boccardo, L. Orsina and I. Peral, A remark on existence and optimal summability of solutions of elliptic problems involving Hardy potential, Discrete Contin. Dyn. Syst., 16, (2006), 513-523.

[8] H. Brezis, L. Dupaigne and A. Tesei, On a semilinear elliptic equation with inverse-square potential, Selecta Mathematica, 11, (2005), 1-7.

[9] H. Brezis and P. Lions, A note on isolated singularities for linear elliptic equations, in Mathematical Analysis and Applications, Mathematical analysis and applications, Part A, pp. 263266, Adv. in Math. Suppl. Stud., Academic Press, New York-London, 1981.

[10] H. Brezis and M. Marcus, Hardy's inequalities revisited, Ann. Sc. Norm. Super. Pisa Cl. Sci., 25, (1997), 217-237.

[11] H. Brezis and L. Nirenberg, Removable singularities for nonlinear elliptic equations, Topol. Methods Nonlinear Anal., 9, (1997), 201-219.

[12] H. Brezis, L. Vázquez, Blow-up solutions of some nonlinear elliptic problems, Rev. Mat. Univ. Complut. Madrid, 10, (1997), 443-469. 
[13] K. Brown, C. Cosner and J. Fleckinger, Principal Eigenvalues for Problems with Indefinite Weight Function on RN, Proc. of the Amer. Math. Soc., 109, (1990), 147-55.

[14] D. Cao and Y. Li, Results on positive solutions of elliptic equations with a critical HardySobolev operator, Methods Appl. Anal., 15, (2008), 81-95.

[15] J. Chabrowski, The Hardy potential and eigenvalue problems, Opuscula Mathematica, 31, (2011), 173-194.

[16] N. Chaudhuri and F. Cîrstea, On trichotomy of positive singular solutions associated with the Hardy-Sobolev operator, C. R. Math. Acad. Sci. Paris, 347, (2009), 153-158.

[17] R. Cignoli and M. Cottlar, An Introduction to Functional Analysis, North-Holland, Amsterdam (1974).

[18] F. Cîrstea, A complete classification of the isolated singularities for nonlinear elliptic equations with inverse square potentials, Mem. Amer. Math. Soc., 227, (2014), no. 1068.

[19] J. Davila and L. Dupaigne, Hardy-type inequalities, J. Eur. Math. Soc., 6, (2004), 335-365.

[20] L. Dupaigne, A nonlinear elliptic PDE with the inverse square potential, J. d'Analyse Mathématique, 86, (2002), 359-398.

[21] M. Fall, Nonexistence of distributional supersolutions of a semilinear elliptic equation with Hardy potential, J. Funct. Anal., 264, (2013), 661-690.

[22] M. Fall and R. Musina, Sharp nonexistence results for a linear elliptic inequality involving Hardy and Leray potentials, J. Inequal. Appl., (2011).

[23] M. Fall, and R. Musina, Hardy-Poincaré inequalities with boundary singularities, Proc. Roy. Soc. Edinburgh Sect. A, 142, (2012), 769-786.

[24] V. Felli and A. Ferrero, On semilinear elliptic equations with borderline Hardy potentials, J. d'Analyse Mathématique, 123, (2014), 303-340.

[25] S. Filippas, L. Moschini and A. Tertikas, Sharp two-sided heat kernel estimates for critical Schrödinger operators on bounded domains, Comm. Math. Phys., 273, (2007), 237-281.

[26] A. García and G. Peral, Hardy inequalities and some critical elliptic and parabolic problems, J. Differential Equations, 144, (1998), 441-476.

[27] M. Ghergu S. Taliaferro, Isolated Singularities in Partial Differential Inequalities, Cambridge University Press, (2016).

[28] N. Ghoussoub and F. Robert, On the Hardy-Schrödinger operator with a boundary singularity, arXiv:1410.1913.

[29] D. Gilbarg and N. Trudinger, Elliptic partial differential equations of second order, Classics in Mathematics, Springer-Verlag, Berlin, (2001).

[30] K. Gkikas, Existence and nonexistence of energy solutions for linear elliptic equations involving Hardy-type potentials, Indiana Univ. Math. J., 58, (2009), 2317-2345.

[31] K. Gkikas and L. Véron, Boundary singularities of solutions of semilinear elliptic equations with critical Hardy potentials, Nonlinear Analysis: Theory, Methods \& Applications, 121, (2015), 469-540.

[32] B. Guerch and L. Véron, Local properties of stationary solutions of some nonlinear singular Schr?dinger equations, Rev. Mat. Iberoamericana, 7, (1991), 65-114. 
[33] K. Ishige, K. Yoshitsu and O. El Maati, The heat kernel of a Schrödinger operator with inverse square potential, arXiv:1602.04172.

[34] P. Lions, Isolated singularities in semilinear problems, J. Diff. Eq., 38, (1980), 441-450.

[35] L. Moschini and A. Tesei, Parabolic Harnack inequality for the heat equation with inversesquare potential, Forum Math., 19, (2007), 407-427.

[36] M. Marcus and P. Nguyen, Moderate solutions of semilinear elliptic equations with Hardy potential, Ann. I. H. Poincaré - Anal. Non Linéaire, 34, (2017), 69-88.

[37] M. Marcus and V. Moroz, Moderate solutions of semilinear elliptic equations with Hardy potential under minimal restrictions on the potential, arXiv:1603.09265.

[38] S. Merchán and I. Peral, Remarks on the solvability of an elliptic equation with a supercritical term involving the Hardy-Leray potential, J. Math. Anal. Appl., 394, (2012), 347-359.

[39] Y. Naito and T. Sato, Positive solutions for semilinear elliptic equations with singular forcing terms, J. Diff. Eq., 235, (2007), 439-483.

[40] L. Schwartz, Théorie des distributions, Hermann, Paris (1973).

[41] D. Smets, A Concentration-Compactness Lemma with Applications to Singular Eigenvalue Problems, J. Funct. Anal., 167, (1999), 463-480.

[42] A. Szulkin and M. Willem, Eigenvalue problems with indefinite weight, Studia Math., 135, no.2, 191-201.

[43] P. Tolksdorf, Regularity for a more general class of quasilinear elliptic equations, J. Differential Equations, 51, (1984), 126-150.

[44] J. Vázquez and L. Véron, Removable singularities of some strongly nonlinear elliptic equations, Manuscripta Math., 33, (1980), 129-144.

[45] L. Véron, Elliptic equations involving Measures, Stationary Partial Differential equations, 1, (2004), 593-712.

[46] Z. Wang and M. Willem, Singular minimization problems, J. Differential Equations, 161, (2000), 307-320. 